\title{
Active control of plasmonic colors: emerging display technologies
}

Kunli Xiong, Daniel Tordera, Magnus Jonsson and Andreas B. Dahlin

The self-archived postprint version of this journal article is available at Linköping University Institutional Repository (DiVA):

http://urn.kb.se/resolve?urn=urn:nbn:se:liu:diva-157274

N.B.: When citing this work, cite the original publication.

Xiong, K., Tordera, D., Jonsson, M., Dahlin, A. B., (2019), Active control of plasmonic colors: emerging display technologies, Reports on progress in physics (Print), 82(2), 024501.

https://doi.org/10.1088/1361-6633/aaf844

Original publication available at:

https://doi.org/10.1088/1361-6633/aaf844

Copyright: IOP Publishing (No Hybrid Option)

http://iopscience.iop.org/ 


\section{Active Control of Plasmonic Colors: Emerging Display Technologies}

Kunli Xiong,' Daniel Tordera, ${ }^{2 \uparrow}$ Magnus P. Jonsson' and Andreas B. Dahlin.*

1 Dept. of Chemistry and Chemical Engineering, Chalmers University of Technology, 41296 Göteborg, Sweden.

2 Linköping University, Dept. of Science and Technology, Laboratory for Organic Electronics, 60174 Norrköping, Sweden.

$\dagger$ Current address: Holst Centre/TNO, High Tech Campus 31, 5605 KN Eindhoven, The Netherlands.

*Corresponding author: adahlin@ chalmers.se 


\begin{abstract}
In recent years there has been a growing interest in the use of plasmonic nanostructures for color generation, a technology that dates back to ancient times. Plasmonic structural colors have several attractive features but once the structures are prepared the colors are normally fixed. Lately, several concepts have emerged for actively tuning the colors, which opens up for many new potential applications, the most obvious being novel color displays. In this review we summarize recent progress in active control of plasmonic colors and evaluate them with respect to performance criteria for color displays. It is suggested that actively controlled plasmonic colors are generally less interesting emissive displays but could be useful for new types of electrochromic devices relying on ambient light (electronic paper). Furthermore, there are several other potential applications such as images to be revealed on demand and colorimetric sensors.
\end{abstract}




\section{Introduction}

Nowadays most people have become entirely dependent on electronic displays because they are superior for spreading information, especially with the help of the Internet and wireless communication. In most countries a lot of people spend many hours every day looking at different kinds of electronic displays, ranging from large TV screens to mobile phones that fit in our pockets, for work or leisure. For instance, considering TV alone, the average American household has more TVs than people and keeps at least one TV on over 8 hours a day (see data for instance from the Minnesota Department of Health). Media consumption in general among adults in the US has increased to over $11 \mathrm{~h}$ per day according to the Q1 2018 Nielsen Total Audience Report (although that number includes audio-only formats).

Displays may be very simple and segmented (e.g. clock digits) or pixelized with the help of transistor arrays. The first pixelized display was the monochrome Cathode Ray Tube (CRT) invented in 1922, followed many decades later by the color CRT in the 1950s. Introducing colors in displays greatly increases the visual appeal and offers the possibility to communicate information more clearly. The first display with reasonably low power consumption, enabling batteries as power sources, was the liquid crystal display (LCD) technology conceived in 1960s.2 The LCD also offered a huge reduction in screen thickness compared to the bulky CRT by the use of liquid crystal cells that are typically on the order of $\sim 100 \mu \mathrm{m}$ thick and enable fast $(\sim 1 \mathrm{~ms})$ switching. Light emitting diode (LED) displays and, more recently, organic LEDs have also become very popular for consumer electronics displays. Notably, LEDs have narrow emission bands and require low power. The conversion efficiency from electric power to emitted intensity is typically between $30-40 \%$ for visible light. ${ }^{3}$ Such high values are in many ways beneficial, but also illustrate that the power consumption of emissive displays is now close to its lower limit. In 
other words, there is little room for further improvement, which forms an important limitation considering that light emission in displays represents most of the power consumption of common electronic devices.

The only alternative to further save energy in information displays is to use reflective displays or "electronic paper" technologies. ${ }^{4}$ Such displays are based on various kinds of electrochromic surfaces that can switch between different coloration states. ${ }^{.}$Since electronic paper does not emit light, the displays rely on ambient illumination (Fig. 1A) and offer excellent visibility under bright conditions (especially in sunlight) as well as higher eye-comfort than emissive displays because of the paper-like appearance. In addition, potentially negative effects from emitted blue light on the circadian rhythm can be avoided. ${ }^{6}$ The world of electronic paper is so far dominated by electrophoretic displays? (EPDs) in which absorbing (black) or reflecting (white) particles are moved vertically by applied voltages. Importantly, EPDs are bistable, i.e. they maintain a static image without consuming power, and they can be bendable. ${ }^{8}$ Other electronic paper technologies include reflective LCDs, where the illuminating back panel is simply replaced by a mirror. Monochromatic reflective LCDs, usually segmented, are often used on simpler electronic devices for displaying numbers and letters. Color versions are much less common due to the considerable loss in absolute reflectivity as well as technical challenges when introducing subpixels, usually red, green and blue (RGB), which can be achieved by different color filters. The same problem with "dull" images (see further below) also applies to EPDs in color which are commercially available from manufacturers (Eink Triton) but generally do not appear in products. The color version of Nintendo Game Boy and certain wrist watches are some of few products utilizing reflective color LCDs, while segmented monochromatic reflective LCDs are very common. An interesting alternative to EPDs and reflective LCDs is the electrowetting display (EWD) which 
was first presented in $2003 .{ }^{9}$ However, although prototypes have been demonstrated, a commercial EWD product is yet to appear. Another technology for reflective color displays is the Mirasol ereader from Qualcomm, ${ }^{4}$ which made it to the market but was later discontinued.

Recently, there has been a remarkable intense interest in using plasmonic nanostructures for color generation and new display technologies. There has been many publications on plasmonic color generation during the last few years. This is arguably somewhat amusing because the concept is definitely not new. Glass decoration by plasmonic nanoparticles has been widely used since the middle ages or even earlier, the prime example being the Lycurgus Cup. ${ }^{10}$ The whole research field of nanoplasmonics started to grow significantly at the end of the previous century in synergy with advancements in fabrication technologies for creating new nanostructures. Stained glassware has often been used as an example of a connection to everyday life when discussing the science of plasmonic colors. Several reviews have already been presented on the topic of plasmonic color generation $^{1,1.2,13}$ and here we only mention this topic briefly in Section 2.

In this review, we will instead focus on emerging research where plasmonics is used to generate colors that can also be actively controlled, i.e. their intensity can be modulated and/or the color can be altered, thereby forming a basis for electronic display technologies. The last few years have seen a very strong increase in number of publications on this topic, as evident from the reference list in this review. 

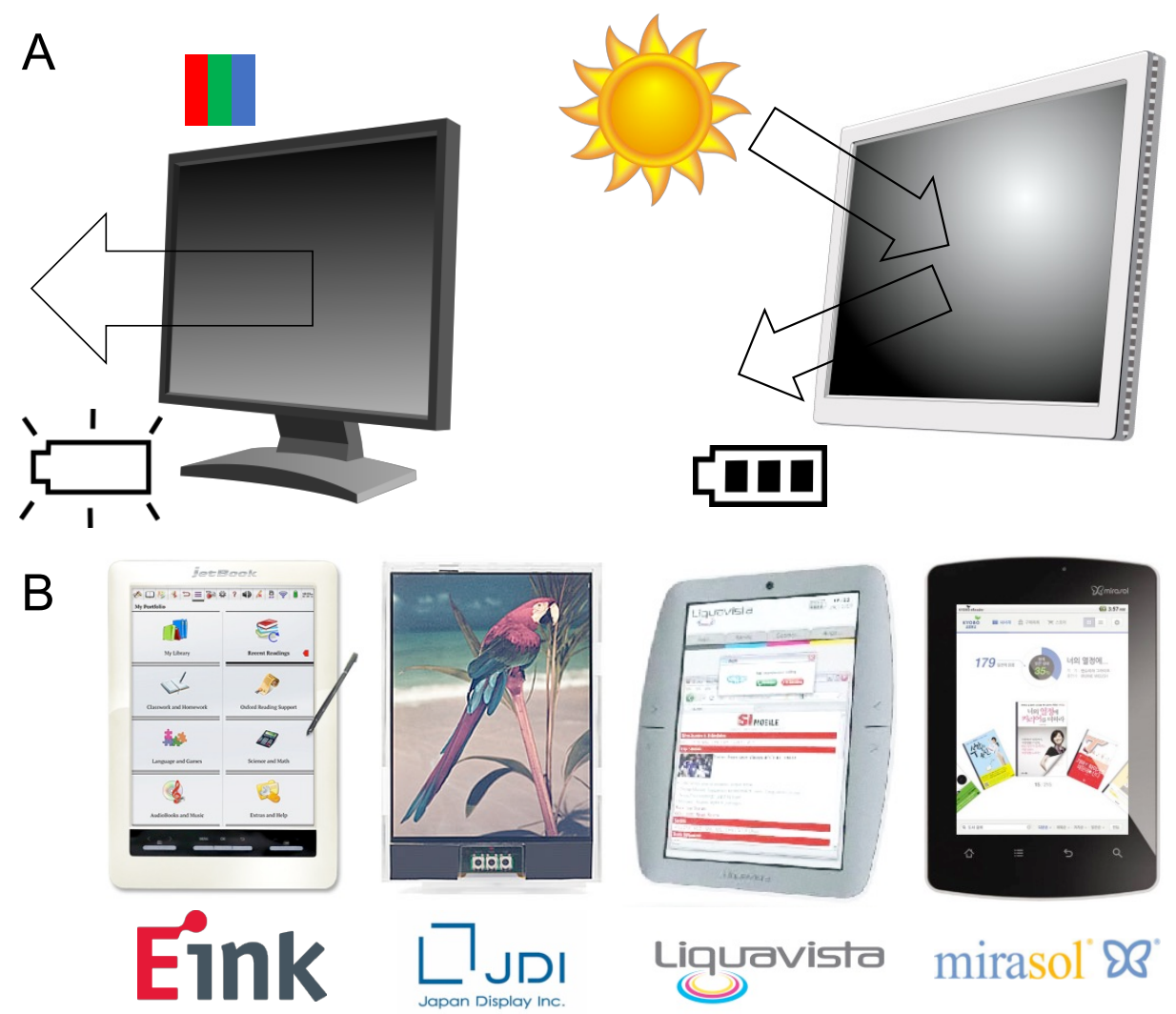

Figure 1. (A) Principles of emissive (left) and reflective displays (right). (B) Examples of color epaper devices from left to right: One type of color EPD using Eink Triton (Ectaco JetBook), a reflective LCD, an EWD device and the Mirasol color e-reader (Qualcomm MEMS Technologies Inc.). At the time of writing, these devices are either prototypes that have not become commercial products or products that were later discontinued. More examples exist. ${ }^{4}$

It should be noted that the definition of "display" in this review is very broad. We consider any active device where colors, generated by plasmonic nanostructures, are somehow tunable in a reversible manner. In other words, the review presents various electrochromic devices based on plasmonics, but it does not aim to cover all "post-processing" techniques that modify the colors in an irreversible manner. As we will see, several devices have so far only presented preliminary results of color tuning and the way to a working fully pixelated or even segmented display is long 
(or in some cases perhaps not reachable). Nevertheless, the techniques may find other applications where actively controlled display of colors is needed, such as counterfeit measures or information display "on demand". The key performance parameters for a display device are summarized in Section 3, which provides an overview of the types of challenges that need to be kept in mind. The "core" of the review is the overview of active concepts for plasmonic color control in Section 4, where we try to relate the presented concepts to the criteria in Section 3. Finally, a few concluding remarks are given in Section 5.

\section{Plasmonic color generation}

A few points regarding the use of plasmonic nanostructures for structural colors are presented here under different subheadings. However, as mentioned, the main part of this review is devoted to dynamic control of colors rather than color generation itself. A full account of recent reports on plasmonic color generation without any form of active tuning or switching is beyond the scope of the current review and the reader is directed to other recent articles..$^{11,1,13,14}$

\subsection{Why plasmonic colors?}

The interest in using plasmonic nanostructures for color generation is manifold. First, due to the strong interaction with light and the subwavelength focus of the field enhancement, very thin layers are sufficient for intense coloration. Many structures are referred to as flat metamaterials or "metasurfaces", ${ }^{15}$ which are typically less than $1 \mu \mathrm{m}$ in thickness. Second, the structures are often stable over long times, at least when compared to organic dyes. This applies in particular to noble metals like gold, but also other metals may be stable if properly enclosed by protective films or inside a glass. The ancient Lycurgus Cup which still shows bright colors contains nanoparticles that consist of not only $\mathrm{Au}$, but also $\mathrm{Ag}$ and $\mathrm{Cu} .{ }^{10}$ Third, it is possible to produce images at extremely high resolution, ${ }^{16}$ even over large areas. ${ }^{17}$ A recent example of structural plasmonic 
coloration over large areas is shown in Fig. 2. Notably, many of these advantages apply to structural colors in general, ${ }^{4}$ not just those generated by plasmonic nanostructures. Dielectric materials are now widely used as an alternative to metals for generating metamaterials with desired optical properties, ${ }^{18}$ including colors, ${ }^{19}$ by utilizing Mie resonances, thin film interference or diffraction from periodic structures. Such structural colors are found in nature, e.g. on butterfly wings s. ${ }^{30}$ The materials are then often lossless, i.e. there is no or very little absorption, only resonant transmission, resonant reflection or directional scattering. However, continuous metal nanostructures can be used as electrodes ${ }^{31}$ and thus offer easier implementation with electronic control for modulating the colors. Also, the strong confinement of plasmonic fields to the metaldielectric surface suggests that the colors are more sensitive to highly localized surface phenomena. 

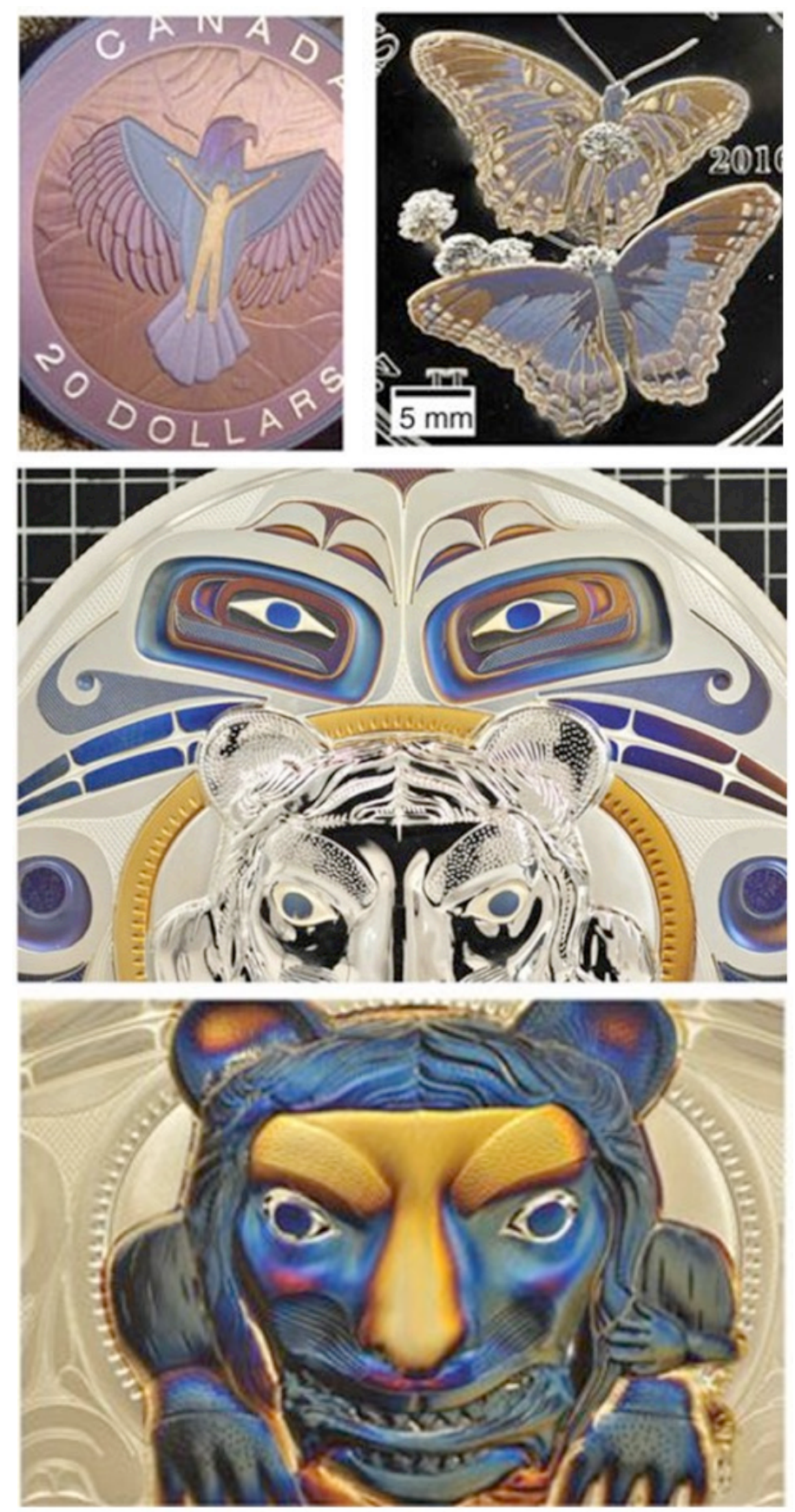

Figure 2. Example of structural plasmonic coloration on metal surfaces. Silver coins were colored by direct exposure to laser pulses creating metal nanoparticles on the surface. (The topographic patterns are already on the coins.) Reproduced from reference Guay et al..$^{20}$ via CC BY 4.0 International License. The images shown here represent a selection from the original article. 


\subsection{Illumination and viewing}

Perceived colors of objects, unless they are non-white light sources, are typically generated by some form of illumination by white light and subsequent selection of certain wavelengths to reach the observer. The perceived colors may depend on the illumination relative to the observer (e.g. "dichroic" glass) and different configurations can be used in a display, regardless of whether it is emissive or relying on ambient illumination. In brief, one can observe transmitted, reflected or scattered light (Fig. 3) or possibly combinations thereof. Backward scattering is equivalent to diffuse reflection, while specular reflection occurs at the same angle relative to the surface normal. As it will be shown, active plasmonic color devices utilize various configurations, although reflection and transmission are the most straightforward. Scattering requires that light is somehow redirected from a source to the observer, which is possible, for example, through nanoparticles on a support by total internal reflection illumination or planar waveguides. ${ }^{21}$ For a display relying on ambient light it is generally complicated to implement scattering mode because ambient light has no defined angle of incidence. However, resonantly scattered light may still contribute to the coloration by not reaching the observer in transmission or reflection mode..$^{22}$ It should also be noted that transmission mode can in principle be used for non-emissive displays if the entire device can be made semi-transparent and placed on an illuminated surface (e.g. a window). 

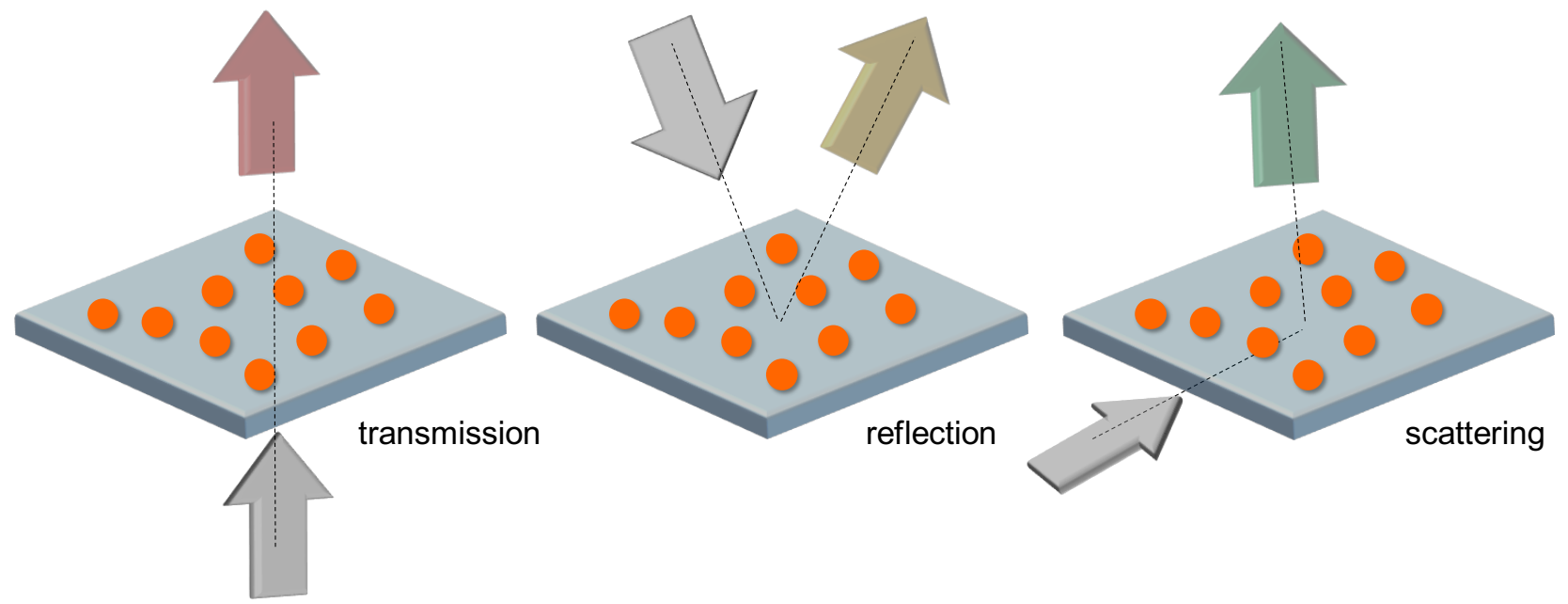

Figure 3. Configurations for displaying plasmonic colors. Reflection may be specular (like a mirror) or diffuse (like paper). The scattering configuration requires dark-field illumination. The particles represent any kind of plasmonic metasurface, i.e. it can also be for instance a nanohole array or more complicated structures. The reflection configuration is normally based on ambient light and the scattering configuration normally requires controlled illumination by a light source.

\subsection{Fabrication and post-processing}

To be visible to the eye, most displays are by necessity covering areas that are very large, at least in the context of nanofabrication where one often prepares highly miniaturized devices. In many cases, the method used for fabricating the plasmonic nanostructures is not compatible with large scales $\left(\mathrm{cm}^{2}\right.$ or more), which means that realizing real display devices requires changing to other methods. This needs to be kept in mind when evaluating the potential of new concepts and devices. Area limitations apply especially for serial techniques such as electron beam lithography (EBL) and focused ion beam (FIB), although laser lithography can be fast enough ${ }^{20}$ For large-area fabrication, lithography based on colloidal self-assembly ${ }^{22.23}$ or chemical methods ${ }^{24,25}$ may be utilized. There are also examples of colorful plasmonic nanostructures that are essentially inkjet printed. ${ }^{26}$ 


\subsection{The subpixel problem}

Emissive displays can normally increase their visibility to a sufficient level simply by emitting more light. However, reflective displays rely on the intensity of the ambient light, which creates a major problem when using subpixels of primary colors to generate secondary colors. For instance, if RGB subpixels are used the total reflectivity goes down by at least a factor of three compared to the reflectivity of a surface consisting of only the primary color. This can be improved by using subtractive colors like cyan, magenta and yellow (CMY) since they represent more of the visible spectrum. In very rough numbers, R, G and B represent wavelength spans of $100 \mathrm{~nm}$, i.e. one third each of the visible range (from approximately 400 to $700 \mathrm{~nm}$ ). In comparison, C, M and Y may be represented quite well by a width closer to $200 \mathrm{~nm}$ each, which typically leads to a higher reflectivity overall compared to RGB. The best solution regarding visibility is, however, to have monopixels instead of subpixels. This means identical pixels where each can generate any desired color. It is clearly more difficult to realize such reflective displays, especially if the pixels should also be able to display black and white. One very challenging option is to construct pixels that somehow contain "vertical" stacks of C, M and Y filters above a mirror, where each stacked layer can be switched independently. ${ }^{4}$

\subsection{Choice of metal}

Plasmonic coloration requires metals with relatively low damping (imaginary part of dielectric function) in the visible so that the resonances are not too broad. A common concern regarding plasmonic coloration is that even the metals with the lowest imaginary part of the permittivity still have considerable loss. Nevertheless, the color quality is clearly sufficient to impress people as evidenced by the historic use of glass stained with metallic nanoparticles. Furthermore, it is not obvious if lossless materials are always preferable, for instance in reflective displays. In spectral 
terms the loss translates into the width of the resonance peaks (or dips). In an emissive display, narrow spectral features are desirable for vibrant colors (see below) and for plasmons the resonance widths are typically around $100 \mathrm{~nm}$, which is more than for an LED. However, wider resonances are suitable for eliminating colors from the visible and for structural coloration based on subtraction. Narrow resonances are important mainly for displays based on scattered or selectively transmitted light (Fig. 3).

Another aspect to consider is cost for fabricating the nanostructure and research has been devoted to using cheap abundant metals, primarily Al, instead of Ag and $\mathrm{Au}$. In other words there has been a shift towards "sustainable plasmonics". ${ }^{27} \mathrm{Al}$ also provides low loss in the UV region. Although the amount of metal in the final device is very low due to the two-dimensionality of the metasurfaces, much is lost in the fabrication process, at least during vacuum deposition steps. ${ }^{28}$ Copper is another interesting option, although it typically requires protective coatings for longterm stability. ${ }^{29}$ This is often the case for $\mathrm{Al}$ as well, but the oxide formed on $\mathrm{Al}$ is not equally bad since it is not lossy, although it may shift the resonances.

\section{Performance parameters for displays}

This section provides an overview of performance indicators for display technologies, which are usually discussed in the context of emissive displays. As many plasmonic color display technologies are passive electrochromic devices we explain how to define the parameters also in such scenarios. We focus on flat-panel displays here, but note that plasmonics is also relevant for development of future projective and holographic display technologies..$^{32}$

\subsection{Brightness}

For emissive displays, brightness is normally defined as luminance (candela per square meter). For reflective displays, the brightness instead translates to the absolute reflectivity of the surface, 
ranging from zero to unity. While image quality for reflective displays will naturally vary with illumination conditions, a high absolute reflectivity, for instance from a plasmonic nanostructure, is vital for good visibility. Since the brightness is wavelength-dependent, proper comparisons require the use of a standardized wavelength. To characterize paper surfaces, blue light at $457 \mathrm{~nm}$ is often used. Paper may have brightness "higher than 100\%" due to addition of fluorescent compounds which absorb in the UV (but the actual reflectivity can never be higher than 100\%). The brightness may be averaged over the visible spectral region but then one should preferably multiply with the luminosity function describing the relative sensitivity of the human eye to different wavelengths. The luminosity function peaks in the middle of the visible but looks slightly different under bright and dark conditions. It is available from the International Commission on Illumination (Commission Internationale de l’Éclairage or CIE).

\subsection{Contrast}

The contrast of a display is defined as the ratio of light intensity (brightness) in the "on" and "off” states. Most devices will operate in a continuum of intensities in which case the contrast is the ratio for the brightest and darkest states. Emissive displays naturally reach extremely high contrast ratios since they can switch off, i.e. any light detected in the off state comes from reflection of ambient light or other pixels. However, there is no strict unifying definition of the parameter due to wavelength dependence, non-standardized lighting conditions and switching dynamics in the intensity. For reflective displays, the difference in reflectivity between on and off states (not the ratio) is often used as a measure of contrast. ${ }^{33,34}$ As an example, a black and white newspaper has a typical contrast of at least $40 \%$ (comparing inked areas with surrounding paper) and the absolute reflectivity of the paper is $\sim 50 \%{ }^{35}$

\subsection{Chromaticity}


Brightness and contrast are important parameters, especially for black and white displays, but they say nothing about the quality of the colors in a display. This is normally defined by an analysis of chromaticity, i.e. how "vibrant" the colors are, a parameter that naturally becomes particularly interesting for plasmonic structural colors. The established standard for evaluating the chromaticity is the CIE diagram from 1931 which is based on a "standard observer" derived from human trials. ${ }^{36}$ In the 2D version of the diagram (Fig. 4) the absolute intensity is normalized and hence it is used to represent chromaticity by coordinates that form a gamut, i.e. a range of accessible colors. The most vivid colors are the monochromatic coordinates along the outer line of the diagram. All secondary colors within the gamut are accessible by combinations of primary (subpixel) colors. For instance, the standard RGB gamut (Fig. 4) generates a triangle and all colors within are possible to produce by varying the relative contributions of $\mathrm{R}, \mathrm{G}$ and $\mathrm{B}$. In the same manner, the (subtractive) CMY colors can also form a gamut covering essentially all colors. As mentioned, $\mathrm{CMY}$ is often beneficial because it increases the overall reflectivity $(\mathrm{C}=\mathrm{B}+\mathrm{G}, \mathrm{M}=$ $B+R, Y=G+R)$. Inkjet printers use CMY colors for higher brightness because the different inks can be stacked upon each other to generate RGB. However, the shape of the CMY gamut in the diagram can make it harder to reach the deep red, green and blue regions (Fig. 4). Note that $\mathrm{C}$ and Y can be located close to the rim of the diagram, i.e. they can be represented by monochromatic light (490 $\mathrm{nm}$ and $580 \mathrm{~nm}$ ), while $\mathrm{M}$ is always a combination of red and blue. 


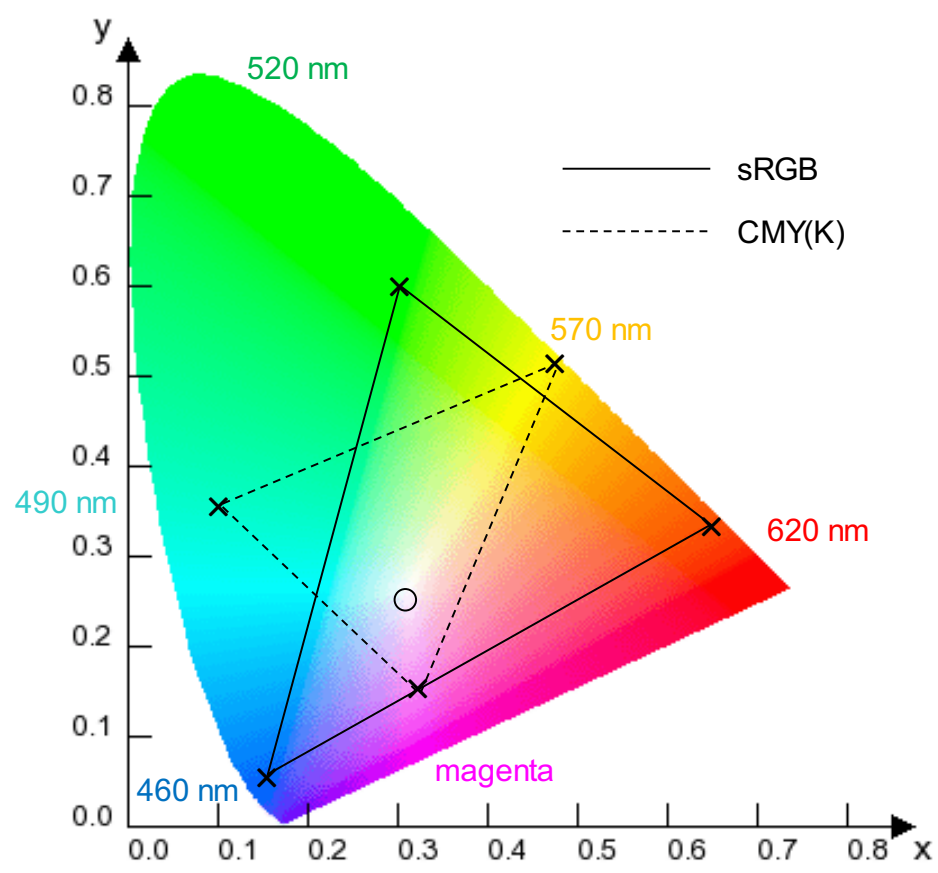

Figure 4. The CIE 1931 xy diagram. Colors are represented by coordinates and can be calculated from the tristimulus values obtained from the color matching functions. Monochromatic light is represented by the outer rim from blue to red. The bottom right magenta border can only be reached by combinations of blue and red. The solid lines show the standard RGB "monitor" gamut, the dotted lines a typical CMY $(\mathrm{K})$ "printing" gamut and the circle is the D65 white point.

Importantly, even if the chromaticity is excellent, i.e. if a wide gamut is covered in the diagram, the brightness can still be too low for the colors to be clearly visible and the contrast can be too low for the display to be useful. In other words, high chromaticity does not imply high brightness and contrast. Indeed, this is a problem often observed in pixelated reflective color displays due to their subpixels (Fig. 1B). For devices relying on ambient illumination, high chromaticity generally occur at the expense of brightness simply because very narrow spectral peaks or dips represent less intensity compared to a broader resonance. In this context, it is interesting to note that plasmonic nanostructures are associated with relatively broad resonance features due to the optical 
loss in metal (as opposed to dielectric structural colors). However, even if broad spectral resonances are associated with lower chromaticity, they can improve the absolute reflectivity by covering a larger part of the visible spectral range. In summary, in many situations plasmonics may provide a good balance between brightness and chromaticity.

\subsection{Response time}

Another important property of displays is the update frequency of the pixels. For smooth video display the response time needs to be around $20 \mathrm{~ms}$ for the pixels, which is easily achieved with LEDs and LCDs, but challenging to reach for many electronic paper technologies (although the EWD is compatible with such switching speeds). As will be shown below, when controlling plasmonic colors the response time varies greatly depending on the mechanism.

\subsection{Resolution}

For pixelated displays, the surface density of pixels determines the resolution of the displayed image. The smallest feature that the eye can resolve at typical handheld reading distance is $\sim 100$ $\mu \mathrm{m}$ which means it is questionable if smaller pixels are necessary. Most fabrication methods can anyhow go well below this value and it is often referred to as an advantage of plasmonic structural colors. The total number of pixels naturally also limits the amount of information that can be displayed.

\subsection{Viewing angle}

Flat panel displays may change appearance depending on the angle between the surface normal and the observer. LEDs do no show such behavior, but for LCDs there is a tradeoff between contrast and viewing angle. Thicker liquid crystals improve the contrast but lead to stronger angular dependence. For reflective displays the viewing angle is sometimes evaluated by specular reflection such that the angle of incidence is defined, but the illumination may also be specified in 
other ways. For plasmonic nanostructures the viewing angle may strongly affect the colors. For instance, nanoparticle dimers may even scatter light of different colors in completely different directions. ${ }^{37}$ The coloration also often depends on how the structure is illuminated and with what polarization, since this can affect the resonance condition, for example when it comes to coupling to propagating modes via periodic structures. ${ }^{38,39,40}$ Angle dependence may be reduced by the use of rougher paper-like surfaces or light diffusers, although at the expense of broadening the spectral features when collecting light over a wider angular interval.

\subsection{Power consumption}

Last but not least, power consumption is a very important performance parameter for displays. It was not merely the bulkiness of CRT screens that made them incompatible with portable devices. When the LCD displays were introduced they also met the requirement of sufficiently low power consumption such that a reasonably small battery was sufficient for quite some usage time, with the consequence that laptop computers started to become common. There are many other examples where power consumption limits the range of possible applications for display devices. The power density is generally calculated as current density multiplied by voltage required. An ultralow power consumption corresponds to less than $10 \mathrm{~mW} / \mathrm{cm}^{2}{ }^{41}$ By comparison, CRT displays consume $>250 \mathrm{~mW} / \mathrm{cm}^{2}$. For electronic paper technologies, low power consumption is often the main factor that motivates research and development since emissive displays provide better performance with respect to the other parameters above. Especially for bistable technologies, the energy consumption can be reduced a lot for static images and the energy density per switch may then become a more relevant parameter. When controlling plasmonic structural colors, the energy required and the degree of bistability varies greatly depending on method as discussed in the next section. 


\section{Active color control}

The following main section of the review summarizes literature on active tuning of plasmonic colors. Some related work will not be discussed in detail. This includes gate-tunable conductive oxides that can change permittivity and induce phase changes for light waves in thin films, but remain limited to the infrared ${ }^{42,43,44}$ To maintain focus of the review, devices where the colors are not based on metallic structures will not be covered, although it should be noted that other interesting electrochromic devices exist, such as amorphous/crystalline phase change materials.$^{45}$ There are also other emerging plasmonic technologies which cannot exactly be classified as dynamic color displays, but certainly closely related, like the plasmonic "liquid mirror" ${ }^{46}$ We present electrochromic devices where the color changes are reversible over at least a few cycles. A useful display device should naturally be stable over many cycles for long-term operation, but even a low number of switching events is considered to be a good start for an emerging technology and such examples are included in this review. It should also be noted that active control of plasmonic colors may here refer to changing from one color to another as well as switching a certain color on and off.

\subsection{Direct electrochemical control}

Electrochemical modulation of plasmon resonances is a relatively old and well-studied field, at least in the context of this review. Some early work by Mulvaney dating back to 1996 showed that the absorption band of small metal clusters could be tuned by electron transfer reactions or chemisorption of ions. ${ }^{47}$ Several studies have later utilized nanoparticles on transparent conductors, mainly indium tin oxide (ITO), in order to tune the plasmon resonance by potential control in a standard three electrode electrochemical configuration, usually with aqueous electrolytes. However, the primary focus has not been electrochromism but rather to develop sensors ${ }^{48}$ or to 
study physiochemical effects on the particles such as heterogeneous catalysis or electron injection into semiconductors. The spectral changes due to applied electrochemical potentials have mainly but not always ${ }^{4}$ been attributed to two effects occurring inside the particles rather than in the surrounding electrolyte: ${ }^{50}$ The first is capacitive charging, which alters the electron density in the metal and thus the effective plasma frequency ${ }^{4,51}$ The second is specific ion adsorption, which creates a lossy optical surface layer. ${ }^{2,23}$ It is not always obvious which effect dominates and under which circumstances, although the type of electrolyte naturally influences the range of possible chemical interactions with ions.

Regardless, the spectral changes due to electrochemical control are generally too small to be useful for generating a reasonable color gamut (typically a few $\mathrm{nm}$ resonance shift) for the voltages used. Increasing the voltages is not an option since this leads to undesired Faradaic reactions which damage the nanostructures. For instance, gold is easily dissolved in the presence of halide ions already above $+0.5 \mathrm{~V}$ vs $\mathrm{Ag} / \mathrm{AgCl}{ }^{.4 ., 5 s}$ Since most studies report resonance shifts and possibly changes in linewidth, it is hard to evaluate what contrasts can be achieved by the devices. However, smaller resonance shifts are associated with smaller changes in absolute transmitted/reflected/scattered intensity unless the resonances are extremely sharp, which as mentioned is not the case for plasmons.

\subsection{Reversible electrochemical deposition}

A more advanced implementation of electrochemistry for control of plasmonic colors is reversible oxidation/reduction of metals on surfaces by potential cycles. Initially, Araki et al. showed that a simple cell consisting of two ITO plates, one flat and one structured, with Ag ions in the sandwiched gel electrolyte, could be used to generate either a transparent state (no metallic Ag), a mirror state (Ag film deposited) or a black state (aggregated Ag particles). ${ }^{56}$ The concept 
was later extended to prepare differently colored surfaces with $\mathrm{Ag} / \mathrm{Cu}$ particles by controlling the nucleation and growth by different voltages, thereby generating colors in the red and blue regions. ${ }^{57}$ The response time was on the order of seconds, which is certainly not fast but not too slow for a useful electrochromic device. Recently, the concept was further developed such that Ag was reversibly deposited on Au "nanodomes" prepared inside small wells..$^{.8}$ This enabled shifting of the particle plasmon resonance throughout the whole visible wavelength range, due to the differences in metal permittivity (Ag resonances are further to the blue due to the higher plasma frequency). By proper design of the core-shell particles, i.e. by controlling the electrodeposition time, the chromaticity was significantly improved. ${ }^{s 8}$ Further, combined with sensory input that analyzed the surrounding colors, a large "plasmonic chameleon" was built (Fig. 5). The electrodeposition method made it possible to switch colors on the timescale of seconds ${ }^{58}$ which may limit the use in displays depending on the application. 

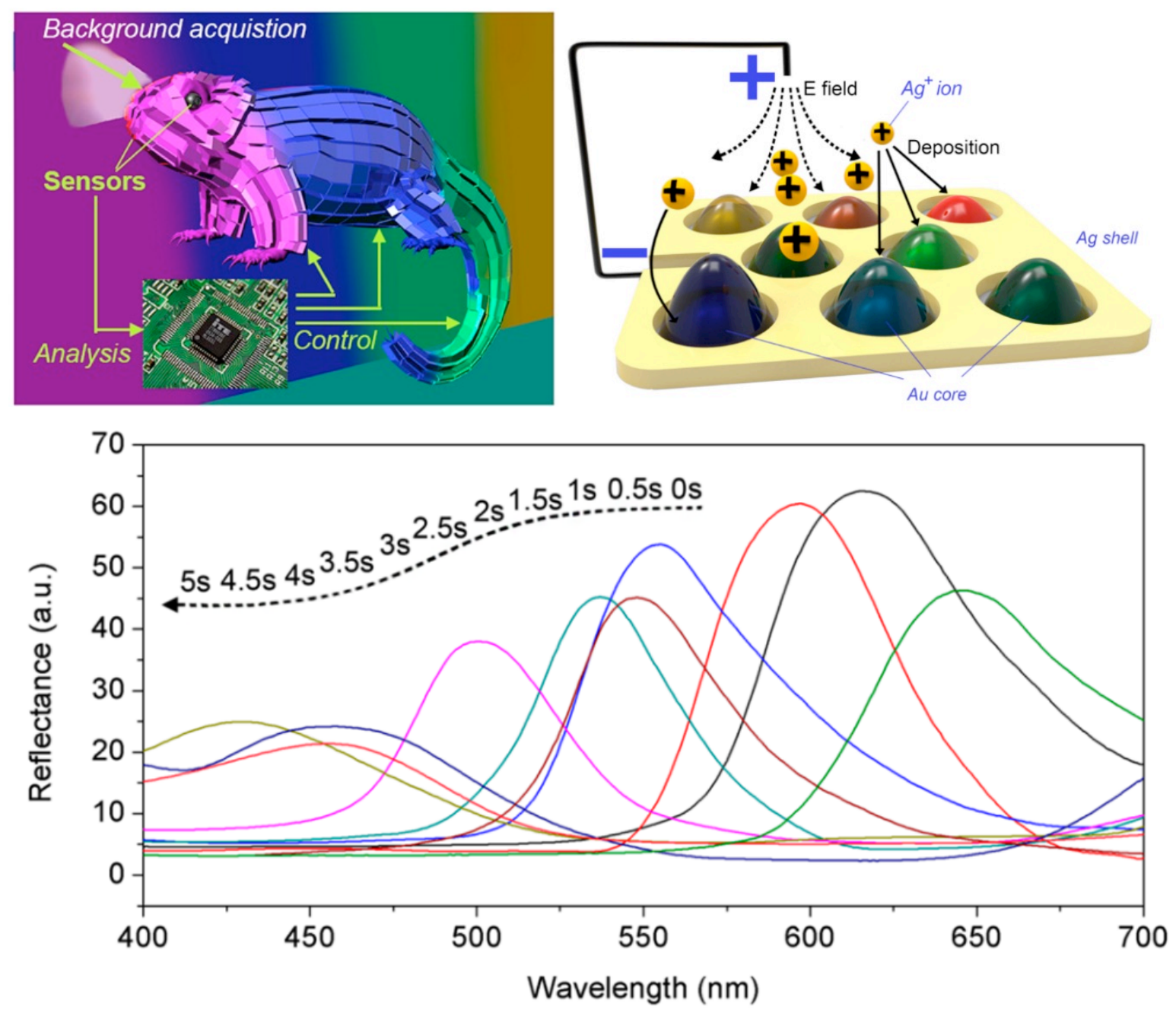

Figure 5. The plasmonic chameleon. Top images show the principle of color sensing and adjustment by reversible electrochemical deposition of $\mathrm{Ag}$ on $\mathrm{Au}$. The bottom shows the reflectivity spectra from the nanoparticle arrays for different deposition times. Adapted with permission from Wang et al. ${ }^{58}$ https://pubs.acs.org/doi/abs/10.1021/acsnano.5b07472 Further permissions related to the material excerpted should be directed to the American Chemical Society.

\subsection{Polarization control}

Several plasmonic color generators, in particular those relying on grating-type excitation of propagating modes, exhibit a dependence on the polarization of the incident light. This is 
especially noticeable for structures consisting of periodically arranged nanoscale apertures in thin metal films. ${ }^{s p}$ Hence color tuning is possible by rotating a polarizer placed in parallel with the nanostructure, preferably by electrical control, ${ }^{\infty}{ }^{\infty}$ even though the nanostructure itself is not altered by this operation. By proper design of the nanostructures, different colors can be generated for different polarizations and thereby the amount of information that can be stored also increases. Heydari et al. presented structures consisting of cross-like apertures on rectangular grids and varied the period in $x$ and $y$ (surface plane) as well as the aperture length in either $x$ or $y{ }^{6 !}$ In this manner, the resonantly transmitted colors could be tuned during fabrication but separately for $x$ and $y$ polarization (Fig. 6A). Al was used as the plasmonic metal. Similar results were presented by Balaur et al. using cross-like apertures in $\mathrm{Ag}$ with rectangular arrangement, i.e. the colors were varying with periodicity but separately in $x$ and $y .2$

Yun et al. presented plasmonic pixels that were covering the whole visible wavelength range with respect to the polarization of the incident light by triplet cavity apertures ${ }^{\star}$ (Fig. 6B). The working mechanism relies on length of the cavity that determines which color is resonantly transmitted through the central aperture. By rotating the polarization of incident light up to $180^{\circ}$, all colors from blue to red could be generated and even secondary colors at intermediate polarizations were achievable (CMY). Note that there was no need to alter any periodicity in $x$ and $y$. Hence the cavity apertures provided full color using a single type of structural entity, i.e. a "monopixel", and are also compatible with high spatial resolution. Some limitations were that the fabrication was based on FIB milling and that the devices were based on resonant transmission which only reached $\sim 1 \%$ in total. 


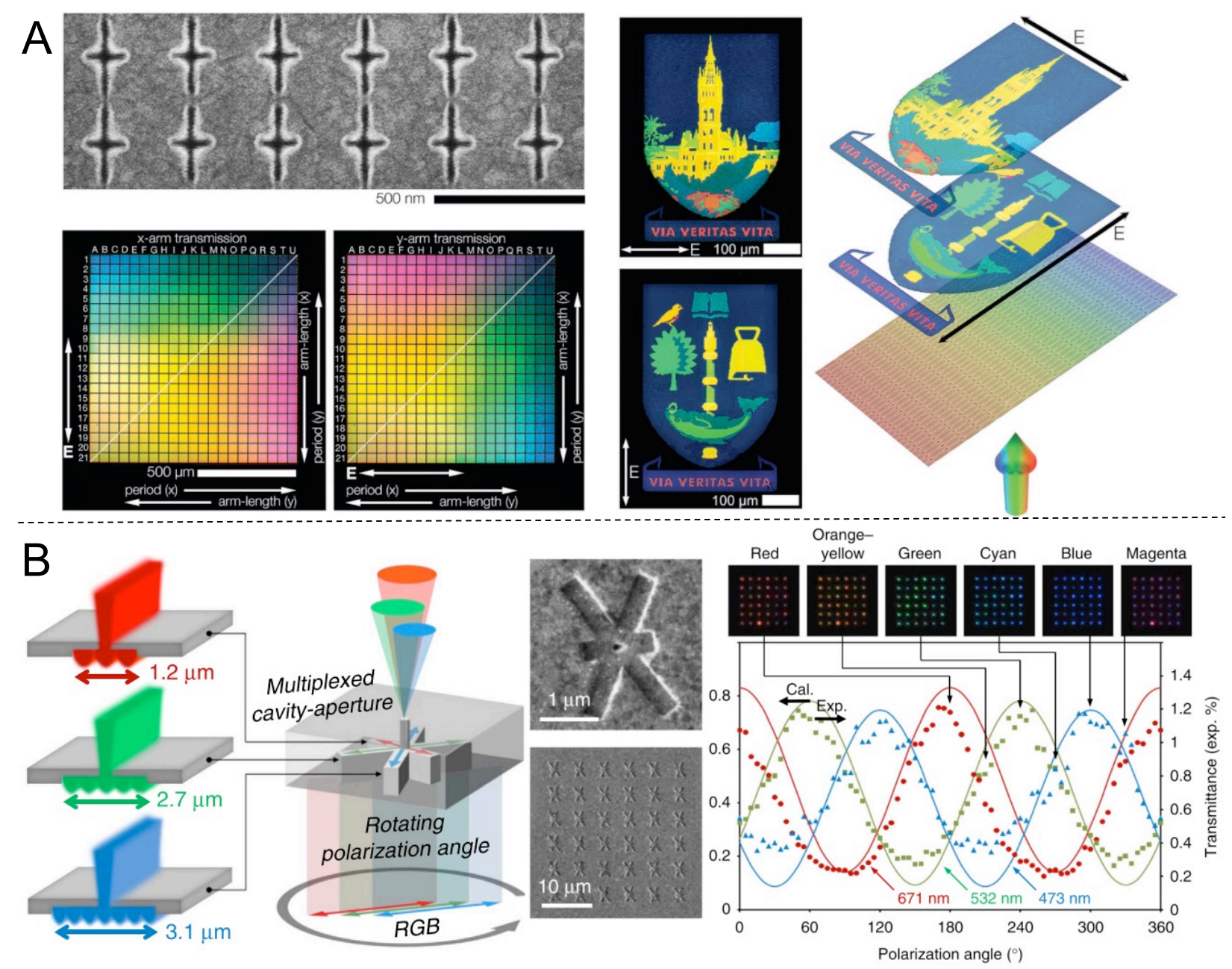

Figure 6. Examples of polarization-chromatic structures which transmit different colors depending on incident polarization. (A) Cross-shaped apertures where two different colors can be encoded on the same area by varying period and aperture length in either dimension. The images on the right have been imprinted onto the same area but each appear under only one polarization. Reproduced from reference Heydari et al. ${ }^{61}$ via CC BY 4.0 International License. (B) Cavity apertures which exhibit full color with polarization rotation from zero to $180^{\circ}$. Red, green or blue light is resonantly transmitted when the polarization is aligned with a cavity long-axis. Reproduced from reference Yun et al..$^{6}$ via CC BY 4.0 International License. 
Duempelmann et al. showed a plasmonic phase retarder based on Ag gratings which could be used to alter the transmitted colors ${ }^{64}$ The plasmonic phase retarder lead to a wavelength-dependent phase shift near the plasmon resonance. As light is transmitted through the nanostructure, it obtains a different polarization depending on wavelength. An analyzing polarizer is introduced after the structure (instead of before). Depending on the angle of the analyzing polarizer, the output colors changed from purple/dark blue to orange/yellow as the analyzing polarizer was set to $0^{\circ}, 45^{\circ}, 90^{\circ}$ or $135^{\circ}$.

\subsection{Liquid crystals for modulating intensity}

Closely related to color tuning by polarization rotation, liquid crystals have been implemented on plasmonic surfaces for dynamic color display in several studies since relatively long. ${ }^{39,65}$ This is not surprising since the technology has been available and it is relatively straightforward to implement for modulation in transmission or reflection mode. Liquid crystals in the vicinity of the nanostructure may in fact alter plasmonic colors in different ways ${ }^{66}$ The most obvious is to simply include a layer of liquid crystals (together with polarizers and electrodes) so that the transmitted or reflected light intensity can be modulated. This enables full color display based on RGB subpixels, but the loss in brightness is severe. This is in part due to the subpixels (section 2.4) but also because the polarizers will only allow $50 \%$ transmission under ambient light. Another concept instead combines liquid crystals with plasmonic colors that depend on polarization (Fig. 6), which is interesting since this reduces or perhaps even eliminates the need for subpixels.$^{63}$ However, the general limitations of LCDs regarding viewing angle would still apply and the plasmonic structures would not offer any advantage in terms of being ultrathin and possibly flexible since a common LCD is anyway relatively thick and not bendable. 
Looking more specifically at certain recent reports in the literature, Franklin et al. showed that electrochromism of nanoimprinted Al structures could be generated merely by a high birefringence liquid crystal which altered the resonant light absorption (Fig. 7A). ${ }^{67}$ The reflectivity was polarization-independent and the coupling to plasmons strongly dependent on the liquid crystal orientation just at the proximity of the surface. The reflectivity peak wavelength could be shifted up to almost $100 \mathrm{~nm}$ by electrical control and the authors proposed that two subpixels (instead of three) could cover all colors by having different periodicities. The viewing angle was reported to be up to $20^{\circ}$ with no strong changes in color. A very important step was taken in subsequent work, where an active matrix was used to address pixels individually, i.e. a pixelated display prototype was demonstrated..$^{68}$ In this follow up work, all colors were accessible by a single nanostructure when varying the voltage and there was no need for subpixels for full color. Some limitations were that a large fraction of the display area was devoted to the supporting electronics and that polarizers were needed in this case since the reflection was polarization-dependent. Both these factors reduce the brightness.

A different approach to realize plasmonic LCDs is to attach the molecules to anisotropic nanoparticles such that they become aligned in a particular direction in an electric field and thereby exhibit a certain color response. ${ }^{9}$ Particles such as gold nanorods may align inside a liquid crystal matrix even if there is no covalent link to the surrounding molecules..$^{70,71, n 2}$ Alternatively, hybrid particles containing magnetically active compounds in addition to the plasmonic metal may align in magnetic fields, but in published work the plasmons appeared in the infrared..$^{3}$

Olson et al. showed an example of plasmonic colors generated from periodic patterns of oriented Al nanorods protected by polyimide..$^{21}$ The nanorods were illuminated in dark field and the scattered colors depended on the edge-to-edge spacing and length of the rods (Fig. 7B). All nanorods were 
oriented in the same direction and far-field effects contributed to narrowing the resonance of the scattered light. By also utilizing Fano interference, the chromaticity of the light scattered collectively from the nanorod array was very high. ${ }^{21}$ For p-polarized light illumination through the substrate it was possible to use liquid crystals to switch the colors off. A twisted nematic cell was used to rotate the emitted light by $90^{\circ}$ such that a subsequent polarizer could block it. One limitation is that the structures were prepared by EBL, which would likely complicate upscaling.
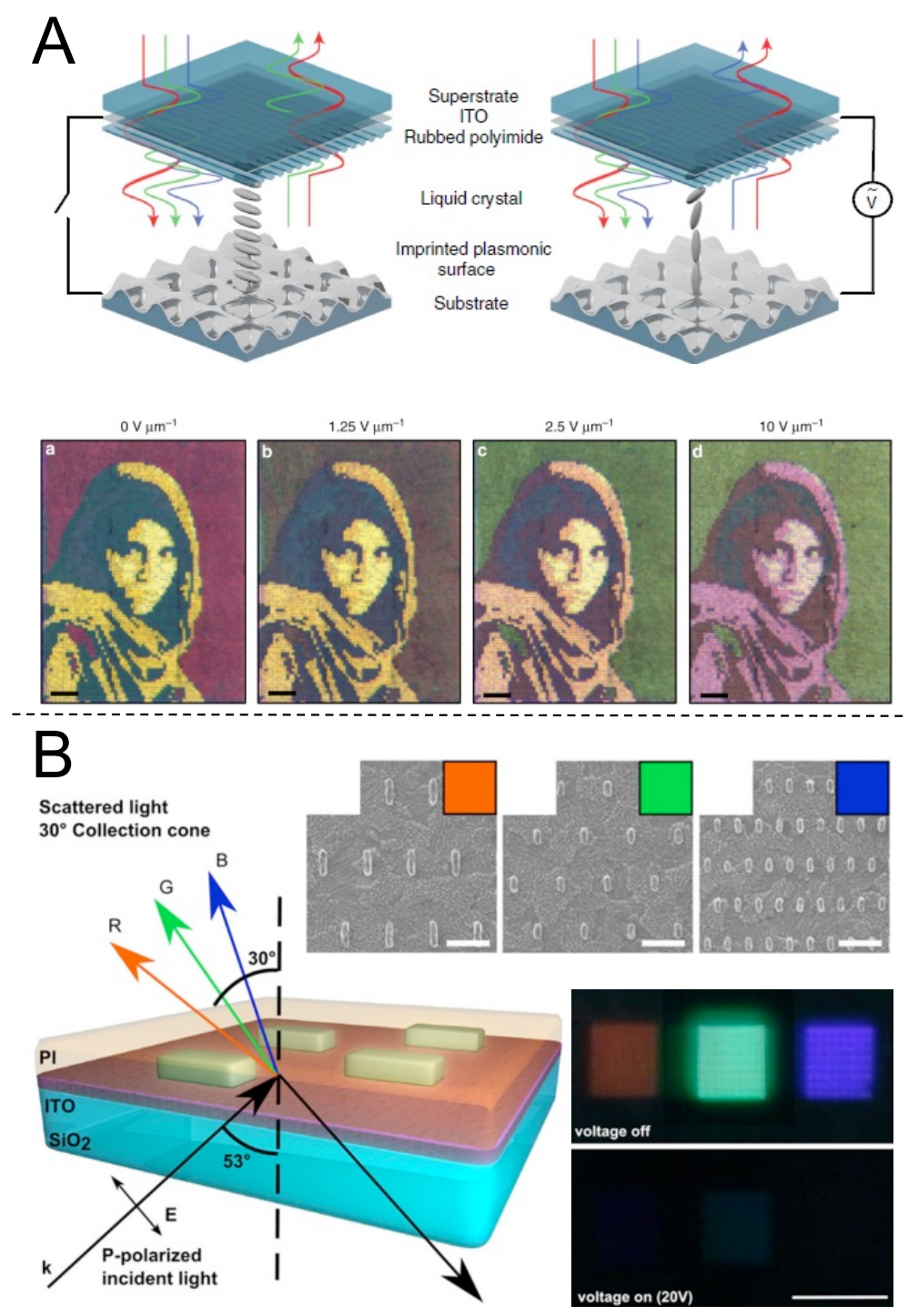
Figure 7. Examples of implementation of liquid crystals. (A) The reflected colors from aluminum nanostructures are tuned by a liquid crystal that changes the resonant light absorption. Reproduced from reference Franklin et al. ${ }^{67}$ via CC BY 4.0 International License. (B) Scattering by arrays of embedded Al nanorods produces red, green or blue light depending on structural parameters. A liquid crystal cell can be used to switch off the emitted colors. Adapted with permission from Olson et al. ACS Nano 10, 1, 1108-1117..' Copyright 2016 American Chemical Society.

\subsection{Conductive polymers for switching colors on/off}

Conductive (or $\pi$-conjugated) polymers have delocalized electrons arising from alternating single-double carbon-carbon bonds in the chain ( $\mathrm{sp}^{2}$ hybridization). In analogy with $\mathrm{p}$ or $\mathrm{n}$ doping of inorganic semiconductors, charge carriers can be introduced to the polymer by oxidation or reduction of the chains. ${ }^{74}$ Besides affecting the electrical properties, this leads to strong changes in the absorption spectrum, normally in the visible or infrared wavelength range. The possibility to repeatedly modulate the oxidation state and thereby the absorption properties has made conductive polymers popular for electrochromic devices and segmented reflective displays. ${ }^{74}$ Pixelized prototypes with passive matrix control have also been demonstrated. ${ }^{75}$ However, the chromaticity of such polymers is usually poor and they are often better at switching from a generally transparent to a generally absorbing state, i.e. they generate a greyscale with voltage. Such black or white "shutters" or "windows" are highly interesting in many applications, ${ }^{33,34,76}$ and if combined with plasmonic coloration they can be used to switch the colors on and off. Hybrid materials consisting of electrochromic polymers on metals are easily created by simple electropolymerization of the conductive nanostructures (for isolated particles an ITO support can be used). Other suitable deposition methods include vapor phase polymerization ${ }^{77}$ and various printing techniques. ${ }^{23}$ 
Functionalizing plasmonic nanostructures with conductive polymers enables plasmonic systems with tunable optical properties. This can be achieved simply through changes in absorption of an overlaying polymer film, which acts as a shutter that regulates the visibility of the colored surface. However, controlling the oxidation state of the polymer can also involve modulation of the plasmonic activity itself, i.e. switching plasmons on and off by controlling the optical damping at the resonance frequency. This has been demonstrated for particles ${ }^{\text {ss }}$ and for resonant transmission through nanohole arrays. .r.79 $^{3.7}$ Resonance shifts induced by applied voltages can also be greatly enhanced ${ }^{80.8,1.22}$ for polymer-functionalized nanoparticles compared to direct electrochemical control of the nanostructures (Section 4.1). Resonance shifts over $100 \mathrm{~nm}$ have been observed when both the particles and the polymer layer have been synthesized in solution, which makes the organic coating fully cover the metal since no solid support is present. ${ }^{83}$ When using the hybridized mode of particle dimers, shifts over $200 \mathrm{~nm}$ have been measured. st $^{\mathrm{s}}$

Although many studies have shown that nanoparticles functionalized with conductive polymers on ITO supports can provide good contrast, ${ }^{7,80,8,8,2,2,8,8,44}$ this configuration only works in transmission mode and the chromaticity is rarely reported. This suggests that modulation of transmission, i.e. optical shutters, rather than dynamic color display been the main motivation for the work. Xu et al. presented a device with improved chromaticity based on nanoslit arrays in $\mathrm{Au}$ or $\mathrm{Al}$, functionalized with polyaniline or poly(ProDOT-Me ${ }_{2}$ ) respectively (Fig. 8A) ${ }^{\text {s5 }}$ The resonant transmission switching was very fast $(10 \mathrm{~ms})$ and the work showed that very thin polymer films are sufficient to generate high contrast in the slit geometry. One limitation is that the devices were prepared by FIB, although the authors suggested that parallel fabrication methods could be implemented in the future. 

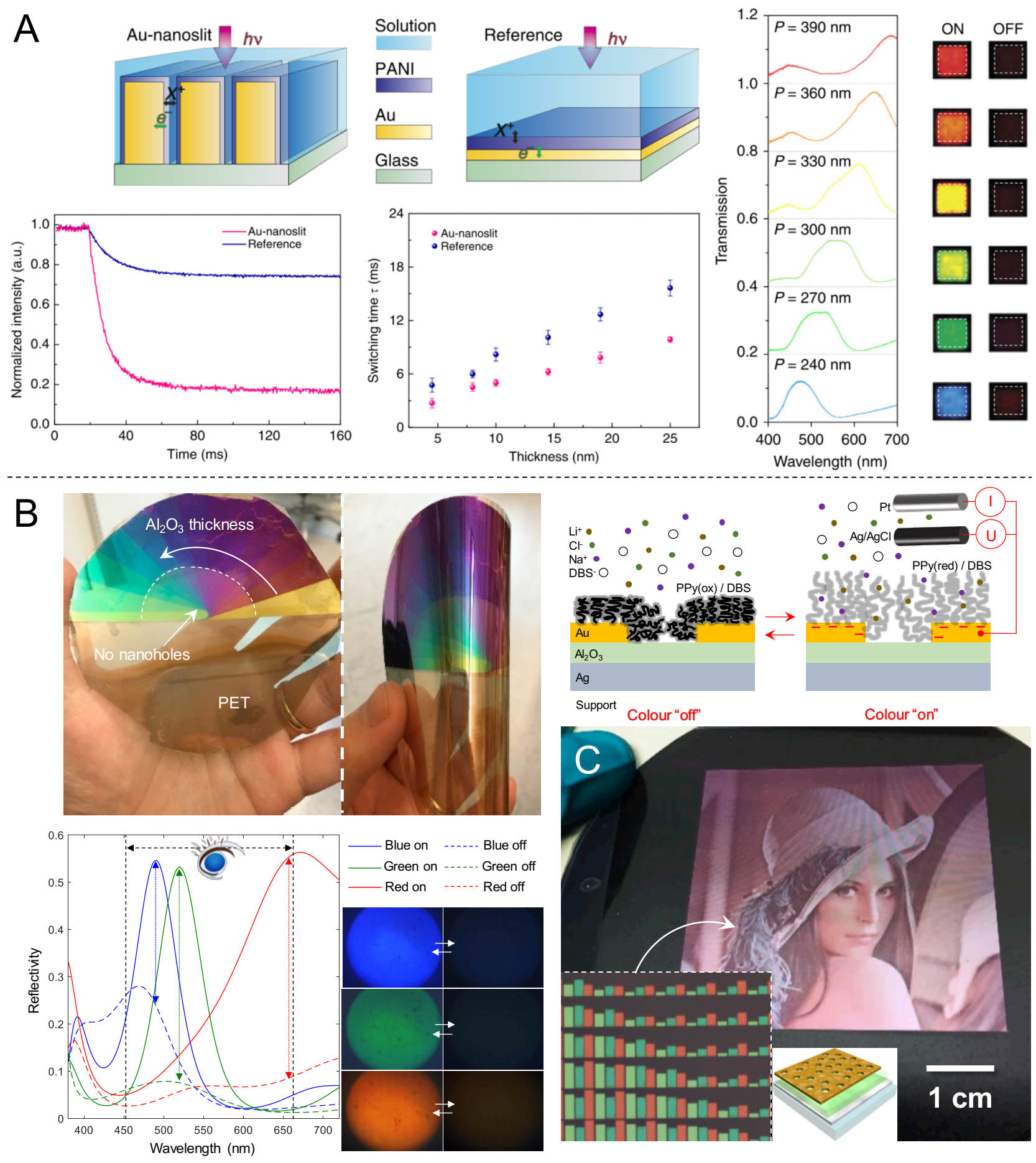

Figure 8. Electrochromism based on conductive polymers on plasmonic nanostructures. (A)

Nanoslits in transmission. Transmission data are for $\mathrm{Al}$ nanoslits with different periods functionalized with poly(ProDOT- $\left.\mathrm{Me}_{2}\right)$. Reproduced from reference $\mathrm{Xu}$ et al. ${ }^{85}$ via CC BY 4.0 
International License. (B) Metasurfaces consisting of an $\mathrm{Ag}$ mirror, an $\mathrm{Al}_{2} \mathrm{O}_{3}$ spacer with variable thickness and a short-range ordered nanohole array in a $20 \mathrm{~nm}$ Au film. The reflectivity spectra show the optical contrast when switching polypyrrole-functionalized samples in an electrolyte. Reproduced with permission from reference Xiong et al. ${ }^{.2}$ Copyright John Wiley and Sons. (C) Nanohole-insulator-mirror metasurfaces patterned by laser lithography into RGB triplets with varying area to reproduce the "Lena" test image. In this sample $\mathrm{Ag}$ was replaced by $\mathrm{Al}$ and $\mathrm{Au}$ was replaced by $\mathrm{Cu}_{.23}$

Xiong et al. presented the first work where plasmonic nanostructures with good chromaticity in reflection mode were combined with conductive polymers, thereby taking the first step towards plasmonic electronic paper..$^{22}$ A short-range ordered nanohole array in $20 \mathrm{~nm}$ Au was used on top of a $\mathrm{Ag}$ mirror and a $\mathrm{Al}_{2} \mathrm{O}_{3}$ spacer and the coloration was a result of thin film interference and surface plasmons excited by the nanoholes, which caused resonant scattering at high angles relative to the surface normal. The fabrication was compatible with large areas as it was based on colloidal self-assembly. Fig. 8B shows an example of a color palette generated by varying the thickness of the $\mathrm{Al}_{2} \mathrm{O}_{3}$ layer, which modifies both the cavity mode reflection peak and the surface plasmon dispersion relation. This picture illustrates the range of colors that can be generated by this nanostructure. In a subsequent study, it was shown that the chromaticity could be equally good even when using abundant metals..$^{23}$ The bottom $\mathrm{Ag}$ mirror was replaced by $\mathrm{Al}$ and $\mathrm{Cu}$ was used for the nanohole array. A thin additional $20 \mathrm{~nm}$ layer of $\mathrm{Al}_{2} \mathrm{O}_{3}$ was sufficient to protect the structures from oxidation in ambient air, at least for several months. By laser lithography, RGB subpixels were written in order to reproduce color images and secondary colors were generated by varying the areas of the RGB triplets (Fig. 8C). This mimics the appearance in a real display device which would be able to vary the reflected intensity in each subpixel. Electrochromic switching was 
performed with polypyrrole (with DBS surfactant) electropolymerized on the gold ${ }^{2}$ and screen printed poly(3,4-ethylenedioxythiophene) polystyrene sulfonate PEDOT-PSS for the case of structures based on $\mathrm{Al}$ and $\mathrm{Cu} \cdot{ }^{23}$ For the optimal thickness of the conductive polymer, high absolute reflectivity (>50\%) was achieved due to the polarization independence as well as good contrast $(>40 \%)$ upon switching the polymer oxidation state. ${ }^{22}$ Similar work on modulating reflected colors using nanowells and polypyrrole was recently presented by Atighilorestani et al. ${ }^{\mathrm{s}} \mathrm{A}$ possible future inorganic alternative to conductive polymers for switching the plasmonic colors is electrochromic metal oxides..$^{87}$

\subsection{Gas adsorption for dynamic color display}

A quite unique approach to make plasmonic colors dynamic was presented by Duan et al. who used hydrogenation of plasmonic Mg nanoparticles to modulate the coloration ${ }^{.8}$ Their main plasmonic component was a $50 \mathrm{~nm}$ thick square-shaped Mg nanoparticle sandwiched between $\mathrm{Ti}$ adhesion layers and a capped layer of $10 \mathrm{~nm} \mathrm{Pd}$. The particles were arranged in square arrays on a $\mathrm{SiO}_{2}$-coated $\mathrm{Si}$ substrate (Fig. 9). As expected, the reflectance from these plasmonic arrays depended on both particle size and interparticle distance. Different combinations of these parameters formed the colour palette shown in Fig. 9. In turn, this enabled pixel-based color production with sub-diffraction limited resolution. The key feature of the system is that the colors can be erased and restored, which is related to the hydrogenation and dehydrogenation of the $\mathrm{Mg}$. When exposed to hydrogen gas, the Pd layer functions as catalyst that dissociates the $\mathrm{H}_{2}$ to atoms. The hydrogen atoms then diffuse into the rest of the particle and transform the $\mathrm{Mg}$ to magnesium hydride $\left(\mathrm{MgH}_{2}\right)$. This metal-to-dielectric phase transition drastically changes the permittivity of the material and hence also the optical properties of the nanoparticle arrays. Indeed, the authors found that the plasmonic colors vanished upon hydrogenation. Moreover, the process is reversible 
and the plasmonic colors and plasmonic images could be restored by dehydrogenation upon exposure to oxygen. The authors explored their dynamic plasmonic concept for secure information encryption. This was achieved by making plasmonic prints for which some areas were insensitive to hydrogen exposure, such that they remained visible and showed up as hidden messages after the active plasmonic areas were hydrogenised and made invisible. The concept was also used for coloration based on cavity resonances between $\mathrm{Al}$ and a thin $\mathrm{Mg}$ film and again image revealing upon exposure to hydrogen. ${ }^{89}$ Recently, a "scanning screen" version of this dynamic color display concept was presented, based on $\mathrm{Al}$ nanoparticles and an underlying Mg support. ${ }^{90}$ 

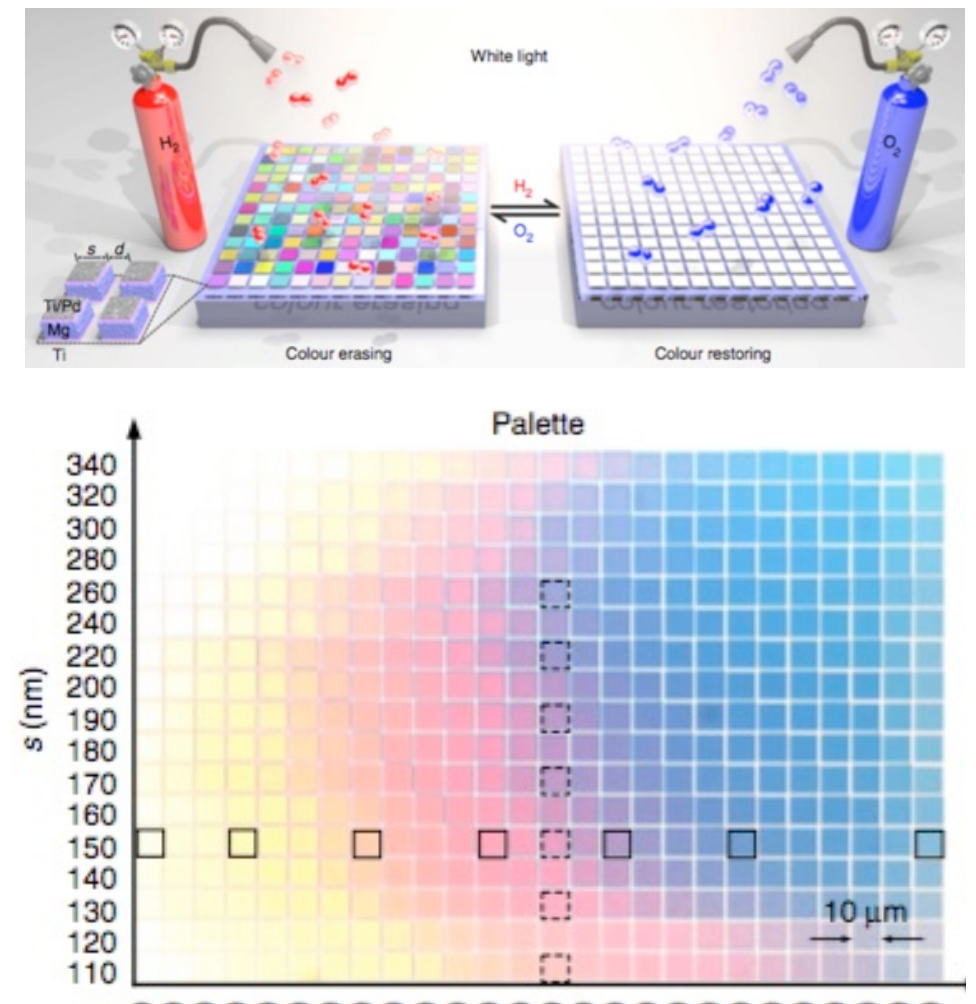

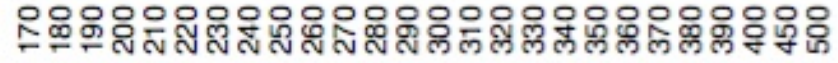

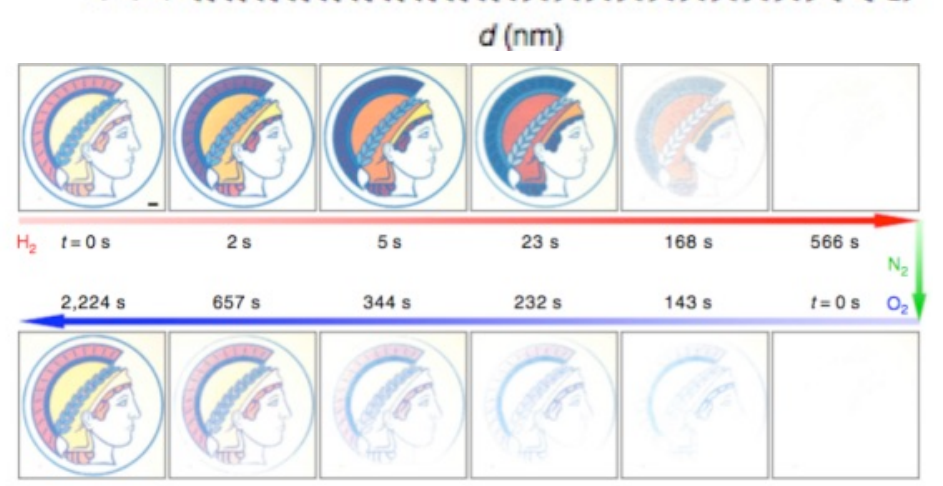

Figure 9. Top image shows a schematic illustration of the concept of reversible dynamic plasmonic surfaces by hydrogenation/dehydrogenation of Mg-based plasmonic surfaces. Middle image shows the color palette obtained by stepwise tuning of particle size $(s)$ and interparticle distance $(d)$. Bottom image shows optical micrographs of the Minerva logo during hydrogenation and dehydrogenation for color erasing and restoring, respectively. Reproduced from reference Duan et al. ${ }^{88}$ via CC BY 4.0 International License. 


\subsection{Electromechanical control of colors}

Nanoscale actuation for active tuning of optical resonances has been a subject of research for a long time. ${ }^{4}$ The principle is based on electrostatic forces induced by applied voltages. In the context of displays it is particularly interesting if one can achieve a resonant reflection which can be tuned across the entire visible wavelength range. This opens up for monopixel reflective displays, which bypass the problem of loss in absolute intensity when using subpixels (Section 2.4), simply because all pixels can show the same color. The most intuitive strategy is to tune a Fabry-Pérot mode by active nanoscale control of the physical distance (optical path length) between two metal films, or the thickness of a cavity between two high index materials. It is noteworthy that such devices have been in development for long and only one was commercialized (the Mirasol technology). ${ }^{4}$ In addition, the monopixels should preferably be able to show black and white.

To date there appears to be relatively few reports on spectral tuning of plasmonic resonances by electromechanical control. One type of electromechanical plasmonic color tuning was reported by Miyata et al. showing how a suspended Au nanowire can be actuated by electrostatic forces for controlling the coupling strength to the underlying metal film.". The voltage needed was $\sim 10 \mathrm{~V}$ and the scattered light was tuned throughout the visible. However, the resonances were broad which indicates low chromaticity and it seems difficult to mass produce the suspended wires and address them individually. Nevertheless, the work shows an interesting new direction for active color modulation because the device is entirely inorganic and solid state. The power consumption was very low and the modulation can go up to $\mathrm{MHz}$ frequencies.9

\subsection{Stretching materials with embedded structures}

Closely related to electromechanical control of plasmonic chromaticity, there have been several reports of purely mechanical actuation, i.e. stretchable plasmonic metasurfaces. The possibility of 
fabricating structural colors on stretchable substrates relies on the fundamental principle of the plasmonic behavior of nanomaterials. An array of nanostructures exhibits one or several characteristic plasmonic peaks. By changing the distance between the elements of the array the peaks can be tuned towards the red or the blue region of the spectrum. This distance can be changed thanks to the elastomeric properties of the substrate, thus allowing creation of different colors by elongating or collapsing the metasurface. Polydimethylsiloxane (PDMS) is usually the substrate material of choice as it possesses very good elastomeric properties, is commercially available and very easy and convenient to work with (inert, non-toxic, non-flammable).

One of the first examples where the elastomeric properties of the substrate were used to tune the plasmonic response can be seen in the work by Pryce et al. showing different types of planar coupled split-ring gold resonators on PDMS. ${ }^{92}$ To characterize their tunable properties they elongated the structures up to $50 \%$, thereby changing the capacitance of the resonators and the coupling strength between pairs. However, this work focused on structures that show plasmonic behavior in the infrared. Song et al. used interference photolithography to create stretchable structures that could be tuned from green to fuchsia..93 Their structures consisted of aluminum nanoparticles deposited on a PDMS substrate. The authors characterized their systems by applying an external force that modulated the period of the structure, causing tuning of the plasmon resonance wavelength. The period changed from $322 \mathrm{~nm}$ at $0 \%$ to $458 \mathrm{~nm}$ at $31.6 \%$ elongation, resulting in a shift of the resonance peak from around $525 \mathrm{~nm}$ to $650 \mathrm{~nm}$. Furthermore, they showed metal-insulator-metal structures with stronger mode coupling. Gao and co-workers prepared arrays of plasmonic structures that could be tuned in a range of almost $600 \mathrm{~nm}$ by applying strains of more than $100 \% .{ }^{94}$ They deposited structures consisting of $40 \mathrm{~nm}$ of either $\mathrm{Au}, \mathrm{Ag}$ or $\mathrm{Al}$ capped by $40 \mathrm{~nm}$ of silicon dioxide with an adhesion layer of $5 \mathrm{~nm}$ of Ti on a PDMS substrate. The analysis 
of the structures showed resonances with a peak of $770 \mathrm{~nm}$ under no strain (period of $300 \mathrm{~nm}$ ). The resonance could be red-shifted all the way to $1310 \mathrm{~nm}$ at $107 \%$ strain. Some work has focused on scalable methods to produce stretchable plasmonic devices. Zhu and co-workers used a selfassembly transfer printing technique to fabricate plasmonic stretchable meta-structures that showed tunable plasmonic behavior with strains up to $38 \% .{ }^{95}$ Yoo et al., ${ }^{96}$ on the other hand, explored template stripping to deposit plasmonic nanostructures on PDMS substrates. This fabrication technique uses a silicon template structured with the inverted pattern of the final structure. The metal is patterned and subsequently transferred to a PDMS substrate to obtain the desired structure. With this technique they fabricated both gold nanohole arrays and gold nanopyramids that, again, showed tunable plasmonic behavior upon stretching of the substrate (with a change of the plasmonic peak from 545 to $682 \mathrm{~nm}$ for just a strain of $9 \%$ in case of the nanopyramids). Finally, they showed how their novel fabrication technique could be applied to nonplanar surfaces by using a cylindrical roller. 

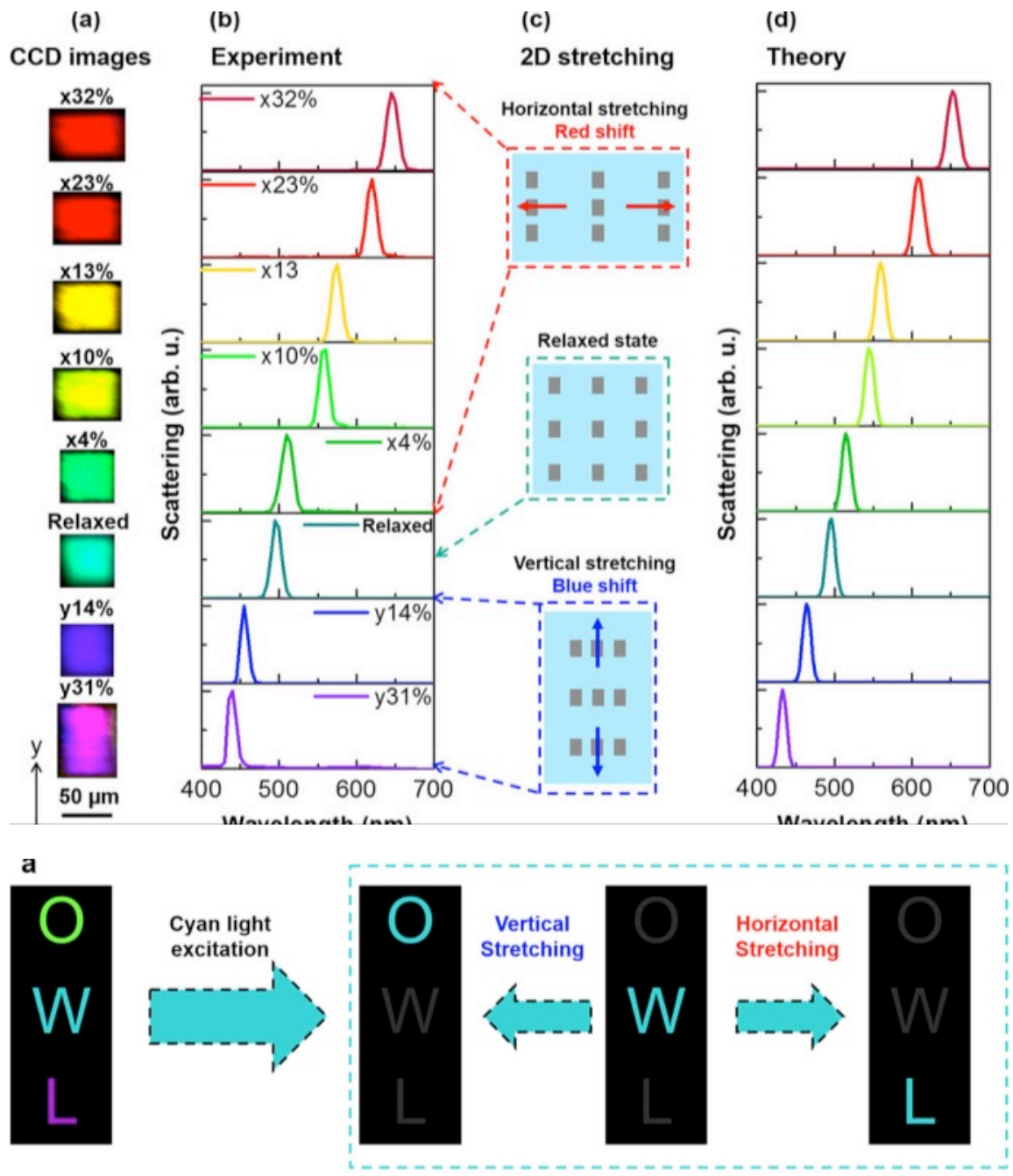

b

C

d
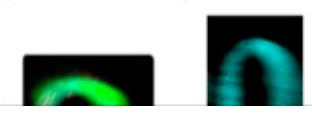

Figure 10. The stretchable plasmonic color display based on arrays of Al particles. Top graphs show experimental and simulated color tuning by stretching in $\mathrm{x}$ or $\mathrm{y}$. The lower images show dynamic revealing and concealing of letters upon stretching. Adapted with permission from Tseng et al. Nano Letters 17, 10, 6034-6039.. Copyright 2017 American Chemical Society.

However, it is not until the work by Tseng et al. ${ }^{40}$ that the first full-spectrum plasmonic color display based on a stretchable substrate was devised. To achieve this they used a square array of $35 \mathrm{~nm}$ aluminum nanostructures with a width of $100 \mathrm{~nm}$, a length of $130 \mathrm{~nm}$ and a period (in $x$ and 
y) of $400 \mathrm{~nm}$ on a PDMS substrate. The structures were designed to exhibit green light in the relaxed state and the color was then modulated by stretching the system, either along the long edge of the nanoparticles ( $y$ axis, blue shift) or along the short edge of the nanoparticles ( $x$ axis, red shift). In the relaxed state the scattering maximum was $495 \mathrm{~nm}$. Applying a strain of up to $32 \%$ shifted the scattering peak to $645 \mathrm{~nm}$ and $440 \mathrm{~nm}$ for the horizontal and vertical stretching, respectively (Fig. 10). With this design, they were able to cover as much as $76 \%$ of the entire CIE diagram, resulting in a chromaticity even better than the sRGB gamut. In their work they also made switchable optical images, utilizing nanoparticle arrays with different periods. Under a white light source each sub-system scatters a different color. By using an incident light with a narrow wavelength range, only the patterns that had a period matching the light could be observed (Fig. 10). The concept of stretchable color tuning is most likely not feasible in an ordinary pixelated display, but could be very interesting for other color display applications. For instance, such materials may act as passive colorimetric strain sensors.

\subsection{Metal-insulator transitions}

A recent study by Shu et al. showed dynamic plasmonic colors by the insulator-metal transition of vanadium oxide. ${ }^{97}$ Structures based on periodic arrays of nanoparticles on thin films containing $\mathrm{VO}_{2}$ showed different colors at $80{ }^{\circ} \mathrm{C}$ compared to room temperature. Although this method for controlling plasmonic colors is too complex and inefficient to implement in a pixelated display it shows yet another example of how one can design a colorimetric indicator which responds to different environmental parameters, in this case temperature.

\section{Discussion}

The previous section has summarized recent developments on dynamic plasmonic color displays. Clearly, many very different concepts have recently been explored to tune plasmonic 
colors and the research field attracts a lot of attention. It is also clear that new types of emissive flat panel displays are perhaps the least likely application since it is overall hard to find any clear benefits from implementing plasmonic nanostructures. In other words, it is hard to find performance parameters (Section 3) where it is clear that plasmonics can make a major improvement. However, this might not be the case for advanced projective and holographic future displays.2. Plasmonic colors themselves may be vibrant but they are not likely to look better than existing technologies in televisions etc. Still, one advantage that may be interesting is that plasmonic colors are generated from very thin metasurfaces, in contrast to glass filters. Having flexible layers may also be interesting, but on the other hand flexible organic LEDs have already been developed to a level where commercial products are beginning to appear. It can be noted, however, that there is clearly a lack of passive or reflective display technologies that operate well in full color. The possibility of introducing fully chromatic monopixels can be beneficial for increasing brightness, but mainly for reflective display devices. ${ }^{68}$ Still, based on the devices available on the market today, electronic paper in full color with high brightness and chromaticity remains a bit of a dream, even if one is willing to sacrifice video speed. Such displays could save a lot of power if they replace emissive displays, for instance when it comes to information spread in public areas. We believe that dynamically controlled plasmonic colors have a high chance to make an impact in the display industry with respect to new types of reflective displays. Naturally, some switching mechanisms will be much more feasible to implement in a real device than other more special methods (e.g. gas adsorption and stretching). For instance, LCDs and electrochromic polymers are already used in monochromatic and/or segmented reflective displays.

Yet there is a much broader range of possible applications of for active control of plasmonic colors than ordinary flat panel displays. One is encrypted information storage where color images 
are revealed under the right conditions. Based on the results presented above this technology seems already available, although the methods for encoding the information (EBL etc.) may be too expensive. Further, plasmonic structures have been proposed as filters in CMOS image sensors. ${ }^{98.9}$ Usually such photodetectors (whether inorganic or organic) are broadband and there is a need for techniques to achieve color selectivity. Letting plasmonic nanostructures act as filters on photodetector pixels opens up for color imaging with high spectral resolution and potentially also at very high spatial resolution. ${ }^{\text {wo, }}$ or Another closely related application to color display is colorimetric indicators of different kinds as simple sensors for the naked eye. Structural colors have already been used as visual indicators of, for instance, different liquids ${ }^{12}$ and plasmonic nanoparticles are already used widely as biological sensors that can give signals visible to the naked eye. ${ }^{103}$ Finally, plasmonic nanostructures may be implemented in "smart" materials that provide simple color indicators of various conditions such as strain (Fig. 10) or temperature. The future of this emerging field will tell.

In conclusion, as we have tried to motivate in this review there are three types of applications which seem to be the most likely: One is new types of high performance reflective displays or electronic paper technologies in color, for which there is still a need on the market. The second is information storage by miniaturized color images which are revealed "on demand", for instance by exposure to specific gases, which could offer new possibilities in counterfeit technologies and secure communication. The third is new types of "smart" materials with colorimetric indicators of temperature or mechanical strain etc. Low cost of production for the plasmonic nanostructures will be an important factor to keep in mind in order to succeed with these applications. 


\section{Acknowledgements}

A.B. Dahlin and M.P. Jonsson acknowledge a recent joint grant from the Swedish Foundation for Strategic Research (application EM16-0002), which inspired us when writing this review and provides support for further research on the topic.

\section{References}

1. Gupta, S.; Sharma, V.; Sharma, P. Cathode ray tube and its applications. Int. J. Innov. Res. Sci. Eng. Technol. 2014, 1 (6), 1731-1733.

2. Williams, R. S. Liquid crystals in an electric field. Nature 1963, 199 (4890), 273-274.

3. Gayral, B. LEDs for lighting: Basic physics and prospects for energy savings. C. R. Phys. 2017, 18 (7), 453-461.

4. Heikenfeld, J.; Drzaic, P.; Yeo, J. S.; Koch, T. A critical review of the present and future prospects for electronic paper. J. Soc. Inf. Display 2011, 19 (2), 129-156.

5. $\quad$ Eh, A. L.-S.; Tan, A. W. M.; Cheng, X.; Magdassi, S.; Lee, P. S. Recent advances in flexible electrochromic devices: the prerequisites, challenges and prospects. Energy Technol. 2017, $6(1), 1-14$.

6. Chang, A. M.; Aeschbach, D.; Duffy, J. F.; Czeisler, C. A. Evening use of light-emitting eReaders negatively affects sleep, circadian timing, and next-morning alertness. P. Natl. Acad. Sci. USA 2015, 112 (4), 1232-1237.

7. Comiskey, B.; Albert, J. D.; Yoshizawa, H.; Jacobson, J. An electrophoretic ink for allprinted reflective electronic displays. Nature 1998, 394 (6690), 253-255.

8. Chen, Y.; Au, J.; Kazlas, P.; Ritenour, A.; Gates, H.; McCreary, M. Electronic paper: Flexible active-matrix electronic ink display. Nature 2003, 423 (6936), 136-136.

9. Hayes, R. A.; Feenstra, B. J. Video-speed electronic paper based on electrowetting. Nature 2003, 425 (6956), 383-385.

10. Freestone, I.; Meeks, N.; Sax, M.; Higgitt, C. The Lycurgus Cup - A Roman nanotechnology. Gold Bull. 2007, 40 (4), 270-277.

11. Hedayati, M. K.; Elbahri, M. Review of metasurface plasmonic structural color. Plasmonics 2017, 12 (5), 1463-1479.

12. Kristensen, A.; Yang, J. K. W.; Bozhevolnyi, S. I.; Link, S.; Nordlander, P.; Halas, N. J.; Mortensen, N. A. Plasmonic colour generation. Nat. Rev. Mater. 2016, 2 (11), 16088.

13. Gu, Y. H.; Zhang, L.; Yang, J. K. W.; Yeo, S. P.; Qiu, C. W. Color generation via subwavelength plasmonic nanostructures. Nanoscale 2015, 7 (15), 6409-6419.

14. Ji, C.; Lee, K.-T.; Xu, T.; Zhou, J.; Park, H. J.; Guo, L. J. Engineering light at the nanoscale: structural color filters and broadband perfect absorbers. Adv. Opt. Mater. 2017, 5 (20), 1700368.

15. Yu, N.; Capasso, F. Flat optics with designer metasurfaces. Nat. Mater. 2014, 13 (2), $139-150$.

16. Kumar, K.; Duan, H.; Hegde, R. S.; Koh, S. C. W.; Wei, J. N.; Yang, J. K. W. Printing colour at the optical diffraction limit. Nat. Nanotechnol. 2012, 7 (9), 557-561. 
17. Zhu, X.; Vannahme, C.; Hojlund-Nielsen, E.; Mortensen, N. A.; Kristensen, A. Plasmonic colour laser printing. Nat. Nanotechnol. 2016, 11 (4), 325-329.

18. Jahani, S.; Jacob, Z. All-dielectric metamaterials. Nat. Nanotechnol. 2016, 11, 23.

19. Ruiz-Clavijo, A.; Tsurimaki, Y.; Caballero-Calero, O.; Ni, G.; Chen, G.; Boriskina, S. V.; Martin-Gonzalez, M. Engineering a full gamut of structural colors in all-dielectric mesoporous network metamaterials. ACS Photonics 2018, 5 (6), 2120-2128.

20. Guay, J. M.; Lesina, A. C.; Cote, G.; Charron, M.; Poitras, D.; Ramunno, L.; Berini, P.; Weck, A. Laser-induced plasmonic colours on metals. Nat. Commun. 2017, 8, 16095.

21. Olson, J.; Manjavacas, A.; Basu, T.; Huang, D.; Schlather, A. E.; Zheng, B.; Halas, N. J.; Nordlander, P.; Link, S. High chromaticity aluminum plasmonic pixels for active liquid crystal displays. ACS Nano 2016, 10 (1), 1108-1117.

22. Xiong, K.; Emilsson, G.; Maziz, A.; Yang, X.; Shao, L.; Jager, E. W. H.; Dahlin, A. B. Plasmonic metasurfaces with conjugated polymers for flexible electronic paper in color. Adv. Mater. 2016, 28 (45), 9956-9960.

23. Xiong, K.; Tordera, D.; Emilsson, G.; Olsson, O.; Linderhed, U.; Jonsson, M. P.; Dahlin, A. B. Switchable plasmonic metasurfaces with high chromaticity containing only abundant metals. Nano Lett. 2017, 17 (11), 7033-7039.

24. Manzano, C. V.; Ramos, D.; Petho, L.; Burki, G.; Michler, J.; Philippe, L. Controlling the color and effective refractive index of metal-anodic aluminum oxide (AAO)-Al nanostructures: morphology of AAO. J. Phys. Chem. C 2018, 122 (1), 957-963.

25. Poplausks, R.; Jevdokimovs, D.; Malinovskis, U.; Erts, D.; Prikulis, J. Variable thickness porous anodic alumina/metal film bilayers for optimization of plasmonic scattering by nanoholes on mirror. ACS Omega 2018, 3 (5), 5783-5788.

26. Jiang, H.; Alan, S.; Shahbazbegian, H.; Patel, J. N.; Kaminska, B. Molding inkjetted silver on nanostructured surfaces for high-throughput structural color printing. ACS Nano 2016, 10 (11), 10544-10554.

27. DeSantis, C. J.; McClain, M. J.; Halas, N. J. Walking the walk: A giant step toward sustainable plasmonics. ACS Nano 2016, 10 (11), 9772-9775.

28. Razza, S.; Castro-Hermosa, S.; Di Carlo, A.; Brown, T. M. Research update: Large-area deposition, coating, printing, and processing techniques for the upscaling of perovskite solar cell technology. APL Mater. 2016, 4 (9), 091508.

29. Susman, M. D.; Feldman, Y.; Bendikov, T.; Vaskevich, A.; Rubinstein, I. Real-time plasmon spectroscopy study of the solid-state oxidation and kirkendall void formation in copper nanoparticles. Nanoscale 2017, 9 (34), 12573-12589.

30. Kinoshita, S.; Yoshioka, S. Structural colors in nature: The role of regularity and irregularity in the structure. ChemPhysChem 2005, 6 (8), 1442-1459.

31. Tordera, D.; Zhao, D.; Volkov, A. V.; Crispin, X.; Jonsson, M. P. Thermoplasmonic semitransparent nanohole electrodes. Nano Lett. 2017, 17 (5), 3145-3151.

32. Kim, I.; Yoon, G.; Jang, J.; Genevet, P.; Nam, K. T.; Rho, J. Outfitting next generation displays with optical metasurfaces. ACS Photonics 2018.

33. Shi, P. J.; Amb, C. M.; Knott, E. P.; Thompson, E. J.; Liu, D. Y.; Mei, J. G.; Dyer, A. L.; Reynolds, J. R. Broadly absorbing black to transmissive switching electrochromic polymers. Adv. Mater. 2010, 22 (44), 4949-4953.

34. Welsh, D. M.; Kumar, A.; Meijer, E. W.; Reynolds, J. R. Enhanced contrast ratios and rapid switching in electrochromics based on poly(3,4-propylenedioxythiophene) derivatives. Adv. Mater. 1999, 11 (16), 1379-1382. 
35. Harrison, V. G. W.; Poulter, S. R. C. The measurement of opacity and reflectivity for printing papers. Brit. J. Appl. Phys. 1950, 1 (Jan), 13-17.

36. Smith, T.; Guild, J. The C.I.E. colorimetric standards and their use. Trans. Opt. Soc. 1931, $33(3), 73$.

37. Shegai, T.; Chen, S.; Miljkovic, V. D.; Zengin, G.; Johansson, P.; Kall, M. A bimetallic nanoantenna for directional colour routing. Nat. Commun. 2011, 2, 481.

38. Atighilorestani, M.; dos Santos, D. P.; Jaimes, R. F. V. V.; Rahman, M. M.; Temperini, M. L. A.; Brolo, A. G. Electrochemical control of light transmission through nanohole electrode arrays. ACS Photonics 2016, 3 (12), 2375-2382.

39. Dickson, W.; Wurtz, G. A.; Evans, P. R.; Pollard, R. J.; Zayats, A. V. Electronically controlled surface plasmon dispersion and optical transmission through metallic hole arrays using liquid crystal. Nano Lett. 2008, 8 (1), 281-286.

40. Tseng, M. L.; Yang, J.; Semmlinger, M.; Zhang, C.; Nordlander, P.; Halas, N. J. Twodimensional active tuning of an aluminum plasmonic array for full-spectrum response. Nano Lett. 2017, 17 (10), 6034-6039.

41. Fernandez, M. R.; Casanova, E. Z.; Alonso, I. G. Review of display technologies focusing on power consumption. Sustainability 2015, 7 (8), 10854-10875.

42. Huang, Y. W.; Lee, H. W. H.; Sokhoyan, R.; Pala, R. A.; Thyagarajan, K.; Han, S.; Tsai, D. P.; Atwater, H. A. Gate-tunable conducting oxide metasurfaces. Nano Lett. 2016, 16 (9), 5319-5325.

43. Michel, A. K. U.; Chigrin, D. N.; Mass, T. W. W.; Schonauer, K.; Salinga, M.; Wuttig, M.; Taubner, T. Using low-loss phase-change materials for mid-infrared antenna resonance tuning. Nano Lett. 2013, 13 (8), 3470-3475.

44. Liu, X. G.; Kang, H.; Yuan, H.; Park, J.; Kim, S. J.; Cui, Y.; Hwang, H. Y.; Brongersma, M. L. Electrical tuning of a quantum plasmonic resonance. Nat. Nanotechnol. 2017, 12 (9), 866871.

45. Hosseini, P.; Wright, C. D.; Bhaskaran, H. An optoelectronic framework enabled by lowdimensional phase-change films. Nature 2014, 511 (7508), 206-211.

46. Montelongo, Y.; Sikdar, D.; Ma, Y.; McIntosh, A. J. S.; Velleman, L.; Kucernak, A. R.; Edel, J. B.; Kornyshev, A. A. Electrotunable nanoplasmonic liquid mirror. Nat. Mater. 2017, 16 (11), 1127-1135.

47. Mulvaney, P. Surface plasmon spectroscopy of nanosized metal particles. Langmuir 1996, 12 (3), 788-800.

48. Dahlin, A. B.; Dielacher, B.; Rajendran, P.; Sugihara, K.; Sannomiya, T.; Zenobi-Wong, M.; Voros, J. Electrochemical plasmonic sensors. Anal. Bioanal. Chem. 2012, 402 (5), 1773 1784.

49. Wang, S. P.; Huang, X. P.; Shan, X. N.; Foley, K. J.; Tao, N. J. Electrochemical surface plasmon resonance: basic formalism and experimental validation. Anal. Chem. 2010, 82 (3), $935-$ 941.

50. Brown, A. M.; Sheldon, M. T.; Atwater, H. A. Electrochemical tuning of the dielectric function of Au nanoparticles. ACS Photonics 2015, 2 (4), 459-464.

51. Hoener, B. S.; Zhang, H.; Heiderscheit, T. S.; Kirchner, S. R.; De Silva Indrasekara, A. S.; Baiyasi, R.; Cai, Y.; Nordlander, P.; Link, S.; Landes, C. F.; Chang, W.-S. Spectral response of plasmonic gold nanoparticles to capacitive charging: morphology effects. J. Phys. Chem. Lett. 2017, 8 (12), 2681-2688. 
52. $\quad$ Byers, C. P.; Hoener, B. S.; Chang, W.-S.; Link, S.; Landes, C. F. Single-particle plasmon voltammetry (spPV) for detecting anion adsorption. Nano Lett. 2016, 16 (4), 2314-21. 53. Hoener, B. S.; Byers, C. P.; Heiderscheit, T. S.; De Silva Indrasekara, A. S.; Hoggard, A.; Chang, W.-S.; Link, S.; Landes, C. F. Spectroelectrochemistry of halide anion adsorption and dissolution of single gold nanorods. J. Phys. Chem. C 2016, 120 (37), 20604-20612.

54. Dahlin, A. B.; Zahn, R.; Voros, J. Nanoplasmonic sensing of metal-halide complex formation and the electric double layer capacitor. Nanoscale 2012, 4 (7), 2339-2351.

55. Sannomiya, T.; Dermutz, H.; Hafner, C.; Voros, J.; Dahlin, A. B. Electrochemistry on a localized surface plasmon resonance sensor. Langmuir 2010, 26 (10), 7619-7626.

56. Araki, S.; Nakamura, K.; Kobayashi, K.; Tsuboi, A.; Kobayashi, N. Electrochemical optical-modulation device with reversible transformation between transparent, mirror, and black. Adv. Mater. 2012, 24 (23), 122-126.

57. Tsuboi, A.; Nakamura, K.; Kobayashi, N. A localized surface plasmon resonance-based multicolor electrochromic device with electrochemically size-controlled silver nanoparticles. Adv. Mater. 2013, 25 (23), 3197-3201.

58. Wang, G.; Chen, X.; Liu, S.; Wong, C.; Chu, S. Mechanical chameleon through dynamic real-time plasmonic tuning. ACS Nano 2016, 10 (2), 1788-1794.

59. Garcia-Vidal, F. J.; Martin-Moreno, L.; Ebbesen, T. W.; Kuipers, L. Light passing through subwavelength apertures. Rev. Mod. Phys. 2010, 82 (1), 729-787.

60. Lee, Y.; Park, M.-K.; Kim, S.; Shin, J. H.; Moon, C.; Hwang, J. Y.; Choi, J.-C.; Park, H.; Kim, H.-R.; Jang, J. E. Electrical broad tuning of plasmonic color filter employing an asymmetric-lattice nanohole array of metasurface controlled by polarization rotator. ACS Photonics 2017, 4 (8), 1954-1966.

61. Heydari, E.; Sperling, J. R.; Neale, S. L.; Clark, A. W. Plasmonic color filters as dualstate nanopixels for high-density microimage encoding. Adv. Funct. Mater. 2017, 27 (35).

62. Balaur, E.; Sadatnajafi, C.; Kou, S. S.; Lin, J.; Abbey, B. Continuously tunable, polarization controlled, colour palette produced from nanoscale plasmonic pixels. Sci. Rep. 2016, 6, 28062.

63. Yun, H.; Lee, S. Y.; Hong, K.; Yeom, J.; Lee, B. Plasmonic cavity-apertures as dynamic pixels for the simultaneous control of colour and intensity. Nat. Commun. 2015, 6, 7133.

64. Duempelmann, L.; Luu-Dinh, A.; Gallinet, B.; Novotny, L. Four-fold color filter based on plasmonic phase retarder. ACS Photonics 2016, 3 (2), 190-196.

65. Kim, T. J.; Thio, T.; Ebbesen, T. W.; Grupp, D. E.; Lezec, H. J. Control of optical transmission through metals perforated with subwavelength hole arrays. Opt. Lett. 1999, 24 (4), 256-258.

66. Shao, L.; Zhuo, X.; Wang, J. Advanced plasmonic materials for dynamic color display. Adv. Mater. 2017, 1704338.

67. Franklin, D.; Chen, Y.; Vazquez-Guardado, A.; Modak, S.; Boroumand, J.; Xu, D.; Wu, S.-T.; Chanda, D. Polarization-independent actively tunable colour generation on imprinted plasmonic surfaces. Nat. Commun. 2015, 6, 7337.

68. Franklin, D.; Frank, R.; Wu, S.-T.; Chanda, D. Actively addressed single pixel full-colour plasmonic display. Nat. Commun. 2017, 8, 15209.

69. Zhang, Y.; Liu, Q. K.; Mundoor, H.; Yuan, Y.; Smalyukh, I. I. Metal nanoparticle dispersion, alignment, and assembly in nematic liquid crystals for applications in switchable plasmonic color filters and e-polarizers. ACS Nano 2015, 9 (3), 3097-3108. 
70. Liu, Q. K.; Senyuk, B.; Tang, J. W.; Lee, T.; Qian, J.; He, S. L.; Smalyukh, I. I.

Plasmonic complex fluids of nematiclike and helicoidal self-assemblies of gold nanorods with a negative order parameter. Phys. Rev. Lett. 2012, 109 (8), 088301.

71. Liu, Q. K.; Cui, Y. X.; Gardner, D.; Li, X.; He, S. L.; Smalyukh, I. I. Self-alignment of plasmonic gold nanorods in reconfigurable anisotropic fluids for tunable bulk metamaterial applications. Nano Lett. 2010, 10 (4), 1347-1353.

72. Liu, Q. K.; Yuan, Y.; Smalyukh, I. I. Electrically and optically tunable plasmonic guesthost liquid crystals with long-range ordered nanoparticles. Nano Lett. 2014, 14 (7), 4071-4077. 73. Zhang, M. L.; Magagnosc, D. J.; Liberal, I.; Yu, Y.; Yun, H.; Yang, H. R.; Wu, Y. T.; Guo, J. C.; Chen, W. X.; Shin, Y. J.; Stein, A.; Kikkawa, J. M.; Engheta, N.; Gianola, D. S.; Murray, C. B.; Kagan, C. R. High-strength magnetically switchable plasmonic nanorods assembled from a binary nanocrystal mixture. Nat. Nanotechnol. 2017, 12 (3), 228-233.

74. Camurlu, P. Polypyrrole derivatives for electrochromic applications. RSC Adv. 2014, 4 (99), 55832-55845.

75. Ersman, P. A.; Kawahara, J.; Berggren, M. Printed passive matrix addressed electrochromic displays. Org. Electron. 2013, 14 (12), 3371-3378.

76. Beaujuge, P. M.; Ellinger, S.; Reynolds, J. R. The donor-acceptor approach allows a black-to-transmissive switching polymeric electrochrome. Nat. Mater. 2008, 7 (10), 795-799.

77. Lawal, A. T.; Wallace, G. G. Vapour phase polymerisation of conducting and nonconducting polymers: A review. Talanta 2014, 119, 133-143.

78. Leroux, Y. R.; Lacroix, J. C.; Chane-Ching, K. I.; Fave, C.; Felidj, N.; Levi, G.; Aubard, J.; Krenn, J. R.; Hohenau, A. Conducting polymer electrochemical switching as an easy means for designing active plasmonic devices. J. Am. Chem. Soc. 2005, 127 (46), 16022-16023.

79. Zhou, Z. W.; Yu, Y.; Sun, N. W.; Mohwald, H.; Gu, P. P.; Wang, L. Y.; Zhang, W.;

Konig, T. A. F.; Fery, A.; Zhang, G. Broad-range electrically tunable plasmonic resonances of a multilayer coaxial nanohole array with an electroactive polymer wrapper. ACS Appl. Mater. Interfaces 2017, 9 (40), 35244-35252.

80. Jiang, N. N.; Shao, L.; Wang, J. F. (Gold nanorod core)/(polyaniline shell) plasmonic switches with large plasmon shifts and modulation depths. Adv. Mater. 2014, 26 (20), 32823289.

81. Leroux, Y.; Lacroix, J. C.; Fave, C.; Trippe, G.; Felidj, N.; Aubard, J.; Hohenau, A.; Krenn, J. R. Tunable electrochemical switch of the optical properties of metallic nanoparticles. ACS Nano 2008, 2 (4), 728-732.

82. Stockhausen, V.; Martin, P.; Ghilane, J.; Leroux, Y.; Randriamahazaka, H.; Grand, J.; Felidj, N.; Lacroix, J. C. Giant plasmon resonance shift using poly(3,4-ethylenedioxythiophene) electrochemical switching. J. Am. Chem. Soc. 2010, 132 (30), 10224-10226.

83. Lu, W.Z.; Jiang, N. N.; Wang, J. F. Active electrochemical plasmonic switching on polyaniline-coated gold nanocrystals. Adv. Mater. 2017, 29 (8), 1604862.

84. Jiang, N.; Ruan, Q. F.; Qin, F.; Wang, J. F.; Lin, H. Q. Switching plasmon coupling through the formation of dimers from polyaniline-coated gold nanospheres. Nanoscale 2015, 7 (29), 12516-12526.

85. Xu, T.; Walter, E. C.; Agrawal, A.; Bohn, C.; Velmurugan, J.; Zhu, W.; Lezec, H. J.; Talin, A. A. High-contrast and fast electrochromic switching enabled by plasmonics. Nat.

Commun. 2016, 7, 10479. 
86. Atighilorestani, M.; Jiang, H.; Kaminska, B. Electrochromic-polymer-based switchable plasmonic color devices using surface-relief nanostructure pixels. Adv. Opt. Mater. 2018, DOI: 10.1002/adom.201801179.

87. Xu, J.; Zhang, Y.; Zhai, T.-T.; Kuang, Z.; Li, J.; Wang, Y.; Gao, Z.; Song, Y.-Y.; Xia, $\mathrm{X} . \mathrm{H}$. Electrochromic-tuned plasmonics for photothermal sterile window. ACS Nano 2018, 12 (7), 6895-6903.

88. Duan, X.; Kamin, S.; Liu, N. Dynamic plasmonic colour display. Nat. Commun. 2017, 8, 14606.

89. Chen, Y.; Duan, X.; Matuschek, M.; Zhou, Y.; Neubrech, F.; Duan, H.; Liu, N. Dynamic color displays using stepwise cavity resonators. Nano Lett. 2017, 17 (9), 5555-5560.

90. Duan, X.; Liu, N. Scanning plasmonic color display. ACS Nano 2018.

91. Miyata, M.; Kaijima, A.; Nagasaki, Y.; Takahara, J. Electromechanically tunable plasmonic nanowires operating in visible wavelengths. ACS Photonics 2016, 3 (12), 2268-2274. 92. Pryce, I. M.; Aydin, K.; Kelaita, Y. A.; Briggs, R. M.; Atwater, H. A. Highly strained compliant optical metamaterials with large frequency tunability. Nano Lett. 2010, 10 (10), 42224227.

93. Song, S. C.; Ma, X. L.; Pu, M. B.; Li, X.; Liu, K. P.; Gao, P.; Zhao, Z. Y.; Wang, Y. Q.; Wang, C. T.; Luo, X. G. Actively tunable structural color rendering with tensile substrate. Adv. Opt. Mater. 2017, 5 (9), 1600829.

94. Gao, L.; Zhang, Y.; Zhang, H.; Doshay, S.; Xie, X.; Luo, H.; Shah, D.; Shi, Y.; Xu, S.; Fang, H.; Fan, J. A.; Nordlander, P.; Huang, Y.; Rogers, J. A. Optics and nonlinear buckling mechanics in large-area, highly stretchable arrays of plasmonic nanostructures. ACS Nano 2015, $9(6), 5968-5975$.

95. Zhu, X. L.; Shi, L.; Liu, X. H.; Zi, J.; Wang, Z. L. A mechanically tunable plasmonic structure composed of a monolayer array of metal-capped colloidal spheres on an elastomeric substrate. Nano. Res. 2010, 3 (11), 807-812.

96. Yoo, D.; Johnson, T. W.; Cherukulappurath, S.; Norris, D. J.; Oh, S. H. Templatestripped tunable plasmonic devices on stretchable and rollable substrates. ACS Nano 2015, 9 (11), 10647-10654.

97. Shu, F.-Z.; Yu, F.-F.; Peng, R.-W.; Zhu, Y.-Y.; Xiong, B.; Fan, R.-H.; Wang, Z.-H.; Liu, Y.; Wang, M. Dynamic plasmonic color generation based on phase transition of vanadium dioxide. Adv. Opt. Mater. 2018, 6 (7), 1700939.

98. Yokogawa, S.; Burgos, S. P.; Atwater, H. A. Plasmonic color filters for CMOS image sensor applications. Nano Lett. 2012, 12 (8), 4349-4354.

99. Zheng, B. Y.; Wang, Y. M.; Nordlander, P.; Halas, N. J. Color-selective and CMOScompatible photodetection based on aluminum plasmonics. Adv. Mater. 2014, 26 (36), 63186323.

100. Chen, T.; Reinhard, B. M. Assembling color on the nanoscale: multichromatic switchable pixels from plasmonic atoms and molecules. Adv. Mater. 2016, 28 (18), 3522-3527.

101. Stewart, J. W.; Akselrod, G. M.; Smith, D. R.; Mikkelsen, M. H. Toward multispectral imaging with colloidal metasurface pixels. Adv. Mater. 2017, 29 (6), 1602971.

102. Burgess, I. B.; Loncar, M.; Aizenberg, J. Structural colour in colourimetric sensors and indicators. J. Mater. Chem. C 2013, 1 (38), 6075-6086.

103. Howes, P. D.; Chandrawati, R.; Stevens, M. M. Colloidal nanoparticles as advanced biological sensors. Science 2014, 346 (6205), 53-62. 